\title{
Hsp90 regulation of fibroblast activation in pulmonary fibrosis
}

\author{
Vishwaraj Sontake, ${ }^{1,4}$ Yunguan Wang, ${ }^{2}$ Rajesh K. Kasam, ${ }^{1,4}$ Debora Sinner, ${ }^{3}$ Geereddy B. Reddy, ${ }^{4}$ \\ Anjaparavanda P. Naren, ${ }^{1}$ Francis X. McCormack, ${ }^{5}$ Eric S. White, ${ }^{6}$ Anil G. Jegga, ${ }^{2}$ \\ and Satish K. Madala' \\ 'Division of Pulmonary Medicine, 'Division of Biomedical Informatics and ${ }^{3}$ Division of Neonatology and Pulmonary Biology, \\ Cincinnati Children's Hospital Medical Center, Cincinnati, Ohio USA. ${ }^{4}$ Department of Biochemistry, National Institute of \\ Nutrition, Hyderabad, Telangana, India. ${ }^{5}$ Division of Pulmonary, Critical Care and Sleep Medicine, University of Cincinnati, \\ Cincinnati, Ohio USA. ${ }^{6}$ Division of Pulmonary and Critical Care Medicine, University of Michigan Medical School, Ann \\ Arbor, Michigan, USA.
}

Idiopathic pulmonary fibrosis (IPF) is a severe fibrotic lung disease associated with fibroblast activation that includes excessive proliferation, tissue invasiveness, myofibroblast transformation, and extracellular matrix (ECM) production. To identify inhibitors that can attenuate fibroblast activation, we queried IPF gene signatures against a library of small-molecule-induced geneexpression profiles and identified $\mathrm{Hsp} 9 \mathrm{O}$ inhibitors as potential therapeutic agents that can suppress fibroblast activation in IPF. Although Hsp90 is a molecular chaperone that regulates multiple processes involved in fibroblast activation, it has not been previously proposed as a molecular target in IPF. Here, we found elevated Hsp90 staining in lung biopsies of patients with IPF. Notably, fibroblasts isolated from fibrotic lesions showed heightened Hsp90 ATPase activity compared with normal fibroblasts. $17-\mathrm{N}$-allylamino-17-demethoxygeldanamycin (17-AAG), a smallmolecule inhibitor of Hsp90 ATPase activity, attenuated fibroblast activation and also TCF- $\beta$ driven effects on fibroblast to myofibroblast transformation. The loss of the Hsp90AB, but not the Hsp90AA isoform, resulted in reduced fibroblast proliferation, myofibroblast transformation, and ECM production. Finally, in vivo therapy with 17-AAG attenuated progression of established and ongoing fibrosis in a mouse model of pulmonary fibrosis, suggesting that targeting Hsp9O represents an effective strategy for the treatment of fibrotic lung disease.

Authorship note: A.G.J and S.K.M. are co-senior authors.

Conflict of interest: The authors have declared that no conflict of interest exists.

Submitted: October 26, 2016 Accepted: January 10, 2017 Published: February 23, 2017

Reference information: JCI Insight. 2017;2(4):e91454. https:// doi.org/10.1172/jici.insight.91454

\section{Introduction}

Pulmonary fibrosis is the final common pathway in adult and pediatric interstitial lung diseases in which the lung becomes irreversibly damaged and scarred due to repetitive injury and progressive interstitial expansion $(1,2)$. Idiopathic pulmonary fibrosis (IPF) is an age-associated fatal fibrotic lung disease, with an annual incidence of 4.6 to 7.4 per 100,000 people and median survival of 3 to 5 years after diagnosis ( 3 , 4). In IPF, fibroblasts exhibit excessive proliferation, invasiveness, myofibroblast transformation, and production of collagen and other extracellular matrix (ECM) proteins in the parenchyma and subpleural areas of the lung (5-8). The lack of knowledge of the molecular regulators that initiate and maintain fibroblast activation is a major obstacle to the development of effective therapies for IPF. Although the actual trigger that initiates and maintains the fibrotic phenotype in IPF lungs remains unknown, repetitive injury of the lung epithelium is thought to play a central role in disease pathogenesis $(9,10)$. Studies over the past decade have suggested that chronic injury and multiple profibrotic signaling events are responsible for the fibroblast activation that results in the formation of scar tissue in IPF (11-13). During injury, fibroblasts play a critical role in repair by directional migration, proliferation, and transformation to myofibroblasts (13-15) However, the role of dysregulated molecular processes of fibroblasts in abnormal healing responses that contribute to the formation of fibrotic lesions and unremitting deposition of ECM in the lung parenchyma remains elusive. Multiple signaling pathways and their downstream targets have been implicated in the persistent activation of fibroblasts that occurs in $\operatorname{IPF}(1,16)$. It has been demonstrated that combinatorial inhibition of 2 or more signaling pathways could result in a synergistic dampening of fibroblast activation and attenuation of pulmonary fibrosis $(17,18)$. In a recent study, we found that inhibition of PI3 kinase 
Table 1. Top-ranked compounds from pharmacogenomics screen based on reciprocal relationship between compound-specific and idiopathic pulmonary fibrosis-specific gene signatures

$\begin{array}{lc}\text { Compound Group } & \text { Compounds } \\ \text { Corticosteroids } & \text { betamethasone, dexamethasone, fludrocortisone, fluticasone, and triamcinolone } \\ \text { Hsp90 inhibitor } & \text { 17-AAG (tanespimycin) } \\ \text { Tyrosine kinase inhibitors } & \text { BIBX-1382, canertinib, dasatinib, and tozasertib } \\ \text { MEK inhibitors } & \text { AS-703026, PD-0325901, and selumetinib } \\ \text { Inositol monophosphatase inhibitor } & \text { L-690330 }\end{array}$

and MAPK pathways together is more effective than that of either alone in attenuating pulmonary fibrosis in mouse models $(18,19)$. Furthermore, nintedanib, an inhibitor of multiple tyrosine kinases that is FDA approved for the treatment of IPF, has been shown to simultaneously target multiple signaling pathways involved in fibroblast activation and pulmonary fibrosis (20). Therefore, identification of a single drug or therapeutic target that impacts more than 1 pathway or cellular process of fibroblast activation could both prevent and reverse fibrosis, leading to improved lung function.

Global gene-expression data are useful for classification and characterization of IPF-specific gene sets that regulate fibroblast processes (e.g., proliferation, migration, invasiveness, and ECM production) involved in IPF pathogenesis. Here, we have taken an unbiased chemical genomics approach to identify drug targets that can alter multiple gene networks involved in IPF-related fibroblast activation. By prescreening more than 1 million diverse small-molecule-driven gene-expression profiles in cells with IPF gene signatures, we identified Hsp90 as a potential therapeutic target for IPF. Hsp90 is a highly conserved molecular chaperone involved in protein folding and stabilization of a variety of client proteins associated with the activation of key signaling pathways and regulatory systems $(21,22)$. The biological activity of Hsp90 depends on its capacity to bind and hydrolyze ATP, which drives the closed conformation of the Hsp90 chaperone that represents its active form $(23,24)$. Studies have demonstrated that inhibition of Hsp90 activity significantly impairs migration and invasion of cancer cells $(25,26)$. An inhibitor of Hsp90 ATPase activity, 17- $N$-allylamino-17-demethoxygeldanamycin (17-AAG) exerts antitumor effects by selectively binding to a conserved binding pocket in the N-terminal domain of Hsp90, inhibiting both ATP binding and ATP-dependent Hsp90 chaperone activity (27). In particular, cytoplasmic Hsp90 interacts with actin and tubulin to stabilize the cytoskeleton and regulate cell motility (28), a key cellular process in the pathogenesis of fibrosis in multiple tissues. In support of this, Hsp90 is a molecule of emerging interest in studies of fibrosis of the lung (29), skin (30), heart (31), marrow (32), prostate (33), joints (34), and kidney (35). A recent study demonstrated overexpression of Hsp90 in the skin that was critical for TGF- $\beta$-driven collagen production and myofibroblast differentiation in systemic sclerosis (30). Pharmacological inhibition of Hsp90 ATPase activity was sufficient to reduce bleomycin-induced dermal fibrosis in Tsk-1 mice, with no toxic effects observed. Furthermore, inhibition of Hsp90 by 17-AAG repressed ECM production in murine renal and liver fibrosis models (35-37); however, the molecular basis for this remains unknown.

Here, we demonstrate that the levels of Hsp90 and associated ATPase activity are elevated in IPF lungs and a mouse model of TGF- $\alpha$-induced pulmonary fibrosis. Our findings describe a potential role of Hsp90 in pulmonary fibrosis via activation of proliferation, motility, fibroblast to myofibroblast transformation, and ECM production. Functional enrichment analysis of gene networks was used to identify the gene targets regulated by Hsp90 in IPF. Further, our in vivo studies established the therapeutic efficacy of 17-AAG as an antifibrotic agent in attenuating established and ongoing pulmonary fibrosis. These data suggest unique mechanisms for the generation of pulmonary fibrosis by Hsp90 and use of 17-AAG in the treatment of pulmonary fibrosis.

\section{Results}

Upregulation of Hsp90-driven gene networks in IPF. To identify novel gene candidates for intervention in IPF, we queried differentially expressed gene (DEG) signatures that are up- or downregulated genes in IPF lungs compared with normal lungs (8), using the Library of Integrated Network-based Cellular Signatures (LINCS) 
A
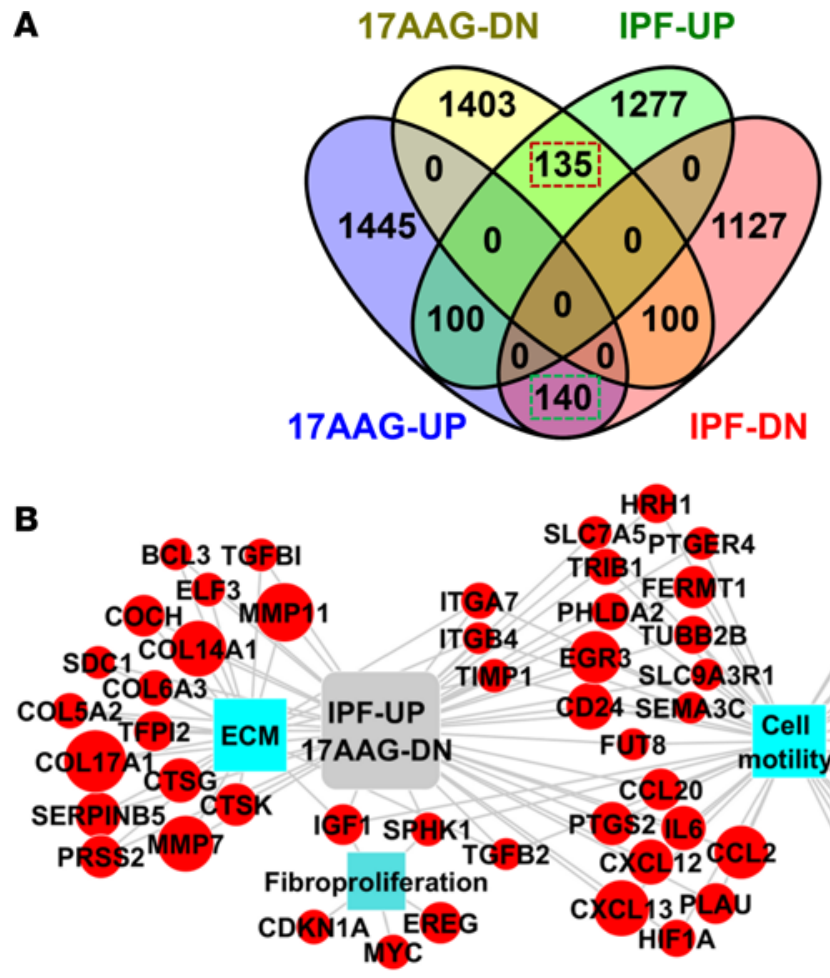

IPF-DN
Figure 1. Hsp90-regulated gene networks in idiopathic pulmonary fibrosis (IPF). (A) Venn diagram depicting the comparison and overlap of differentially expressed genes in IPF lungs and 17-AAG treatment (from LINCS). Of the 2,879 genes differentially expressed in IPF lungs, 475 genes overlapped with differentially expressed genes following 17-AAC treatment. The box indicates genes that are up- (135 genes) or downregulated (DN, 140 genes) in IPF lungs compared with 17-AAG treatment. (B) Hsp90driven gene networks that are activated in IPF were analyzed using ToppFun and visualized using Cytoscape. Red- and green-colored circles represent genes that are up- and downregulated respectively in IPF lungs. The blue-colored squares represent enriched biological processes for the inversely correlated genes between Hsp90 treatment and IPF.

database (http://www.lincscloud.org/). The LINCS library has gene-expression profiles induced by over 20,000 compounds, shRNAs, and kinase inhibitors using the L1000 platform. We used connectivity mapping (implemented as LINCS cloud query app) to identify candidate therapeutics that are reciprocally connected between L1000 perturbagens and IPF (38).

Among the top-ranked small molecules whose gene-expression profiles were inversely correlated to the IPF DEG profile was 17-AAG (also known as tanespimycin), a derivative of the antibiotic geldanamycin and a known inhibitor of Hsp90 ATPase activity (Table 1). Other major compound groups identified through a connectivity-map approach include corticosteroids, tyrosine kinase inhibitors, MEK inhibitors, and an inositol monophosphatase inhibitor. To elucidate the potential mechanism of action of 17-AAG in IPF, we compared the respective gene-expression profiles of 17-AAG (from LINCS) to that of IPF and identified multiple transcripts that were either up- (135 genes) or downregulated (140 genes) in IPF lungs (Figure 1A). We performed an enrichment analysis of these negatively correlated gene sets (i.e., genes upregulated in IPF, but downregulated by 17-AAG and vice versa) using the ToppFun application of the ToppGene Suite (39) (Figure 1B). Notably, among the top enriched biological processes were fibroblast motility, proliferation, growth, and ECM production (Figure 1B and Supplemental Table 1; supplemental material available online with this article; https://doi.org/10.1172/jci.insight.91454DS1).

Increased immunostaining and activity of Hsp90 in fibrotic lungs. To validate Hsp90 as a candidate gene in pulmonary fibrosis, we assessed the levels of Hsp90 by coimmunostaining IPF and normal lung tissue sections with antibodies against Hsp90 and vimentin. The expression of Hsp90 was detected in human lung cells, including in vimentin-positive fibroblasts of normal and IPF lungs (Figure 2A). Notably, the staining of Hsp90 was increased in the lung sections of human IPF compared with normal lungs. Furthermore, images taken at high magnification demonstrated that Hsp90 was localized in both the cytosol and nucleus of fibroblasts in IPF and non-IPF lungs. Also, we observed prominent Hsp90 immunoreactivity in pneumocytes, airway epithelium, and fibroblast foci in areas of active fibrosis in IPF lungs (Figure 2A).

Hsp90 has been shown to attain an active state upon formation of a complex with other cochaperones, a state in which Hsp90 exhibits increased ATPase activity (40). To determine the levels of Hsp90 in pulmonary fibrosis, the lung lysates of control mice and TGF- $\alpha$-transgenic (TGF- $\alpha-\mathrm{Tg}$ ) mice induced on doxycycline (Dox) for 6 weeks were immunoblotted with antibodies against Hsp90. The levels of Hsp90 were heightened in the fibrotic lungs of the TGF- $\alpha-\operatorname{Tg}$ mice compared with the lungs of control mice (Figure 2, B and C). Further, Hsp90-specific ATPase activity was quantified in the lung lysates of control mice and TGF- $\alpha-\mathrm{Tg}$ mice induced on Dox for 6 weeks. We observed a 2-fold increase in Hsp90 ATPase activity in the lung lysates of fibrotic mice compared with normal mice (Figure 2D). Notably, we observed a significant increase in Hsp90 ATPase activity in the lysates of fibroblasts from IPF lungs compared with fibroblasts from normal lungs (Figure $2 \mathrm{E}$ ). To determine whether Hsp90 isolated from fibrotic lungs had a higher binding affinity for 17-AAG 


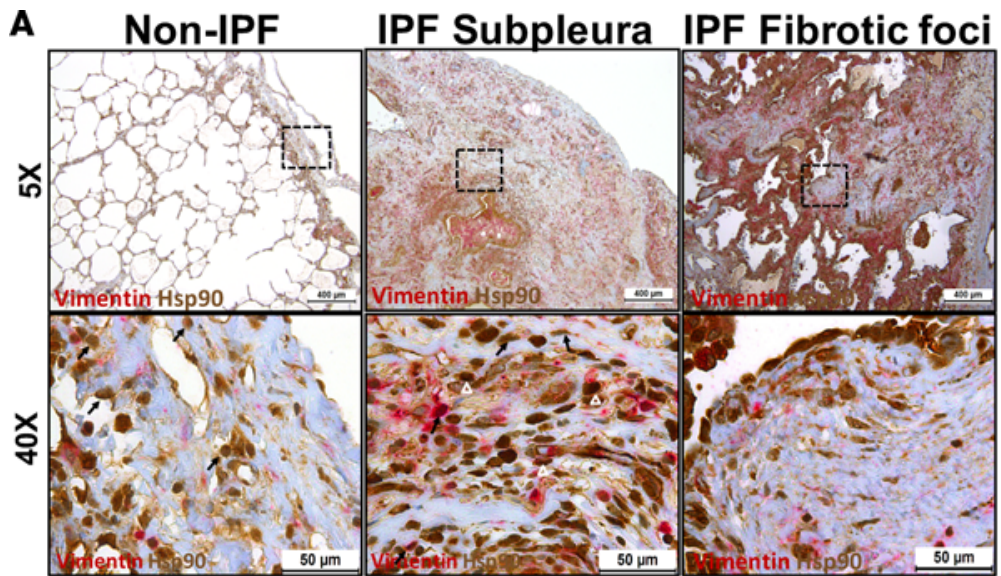

B

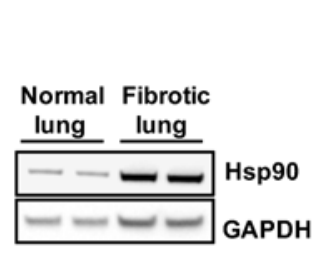

E

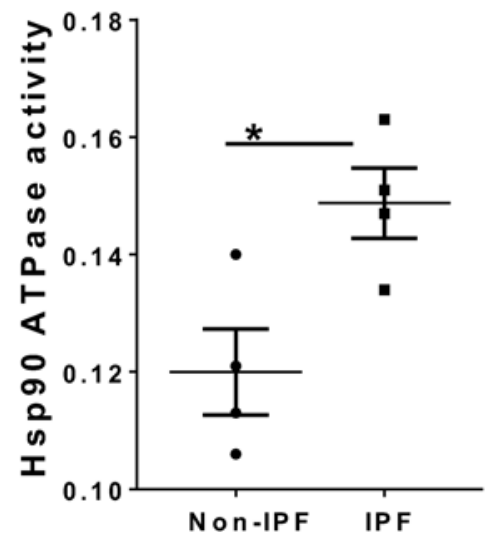

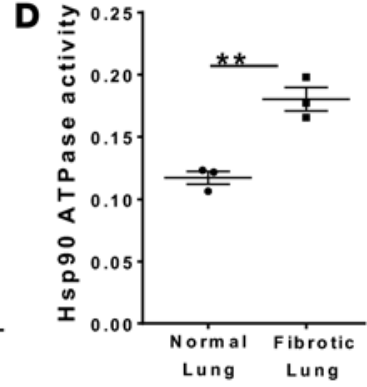

$\mathbf{F}$

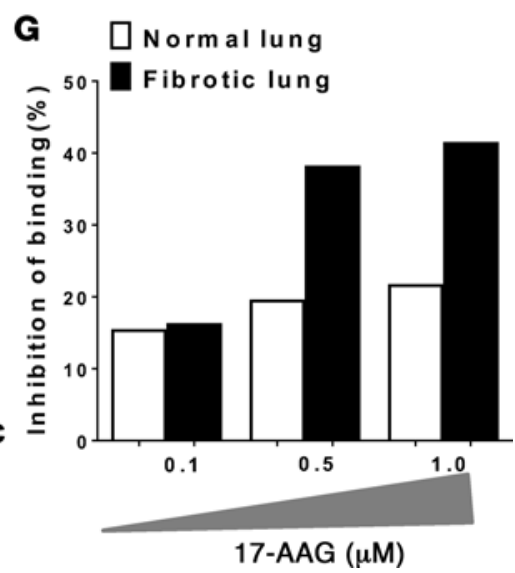

Figure 2. Hsp90 and its ATPase activity elevated in fibrotic lungs. (A) Idiopathic pulmonary fibrosis (IPF, $n=4)$ and non-IPF ( $n=6$ ) lung tissue sections immunostained with antibodies against $\mathrm{Hsp90}$ (brown) and vimentin (purple). All images obtained at low $(\times 5$; scale bars $=400 \mu \mathrm{m})$ and high $(\times 40$; scale bars $=50 \mu \mathrm{m}$ ) magnification. Dashed boxes indicate the area of the lung shown in high-magnification images. Arrows indicate Hsp90 staining in the cytoplasm and nucleus of vimentin-positive lung cells. Hsp9o localization was observed in epithelial cells and vimentin-positive cells of fibrotic foci. (B) The lung lysates immunoblotted with antibodies against Hsp90 to measure increases in Hsp90 in the fibrotic lungs of TCF- $\alpha$-transgenic (TCF- $\alpha$-Tg) mice compared with control mice on doxycycline (Dox) for 6 weeks. (C) Quantification of Hsp9o levels using Phosphor Imager software, and the amount of Hsp90 normalized with GAPDH levels in the lung lysates of TCF- $\alpha$-Tg mice compared with control mice on Dox for 6 weeks ( $n=2 /$ group). (D) Hsp90 ATPase activity measured in the lung lysates of control or TCF- $\alpha-T g$ mice on Dox for 6 weeks ( $n=3 /$ group). (E) Hsp90 ATPase activity measured in the lysates of fibroblasts isolated from IPF and non-IPF lungs ( $n=4$ /group). (F) Hsp90 binding affinity towards 17-AAC was evaluated in a competitive binding assay using a biotinylated geldanamycin (biotin-CM) probe and increasing concentrations of 17-AAG in the lung lysates of control mice or TCF- $\alpha$-Tg mice on Dox for 6 weeks. (C) The percentage inhibition of biotin-GM binding to Hsp9O with increasing doses of 17-AAG in lysates isolated from fibrotic lungs compared with normal lungs. The above results are representative of 2 to 3 independent experiments and reported as mean \pm SEM. An unpaired 2 -tailed Student's $t$ test was performed to measure the significance. ${ }^{*} P<0.05,{ }^{* *} P<0.005$, and ${ }^{* *} P<0.0005$.

than that from normal lungs, we incubated Hsp90 from the lysates of lung fibroblasts with increasing concentrations of 17-AAG in the presence of biotinylated geldanamycin (Figure $2 \mathrm{~F}$ ). Increasing doses of 17-AAG resulted in a decrease in binding of Hsp90 to the geldanamycin, which was greatest with $\mathrm{Hsp} 90$ of fibroblasts isolated from the fibrotic lungs of TGF- $\alpha-\mathrm{Tg}$ mice compared with normal mice (Figure 2, F and G). 
A

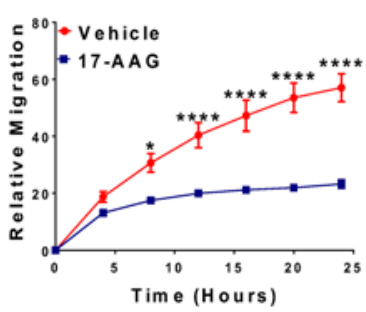

D

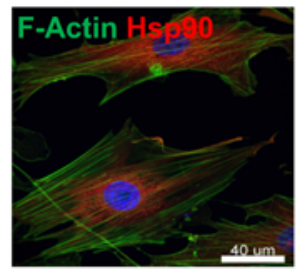

B

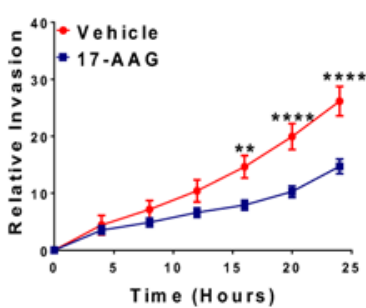

C

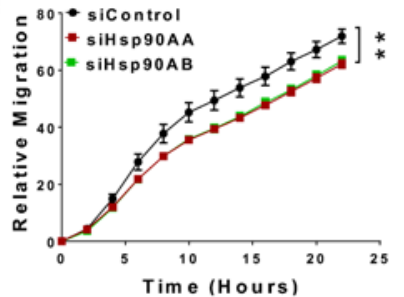

E
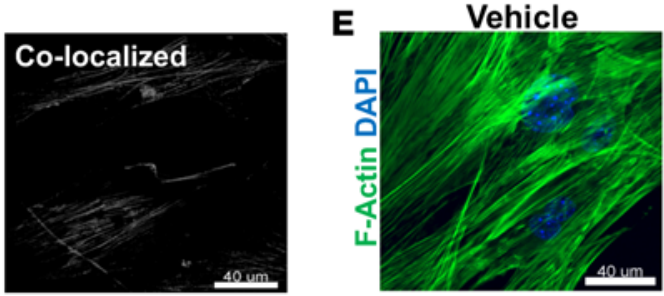

17-AAG
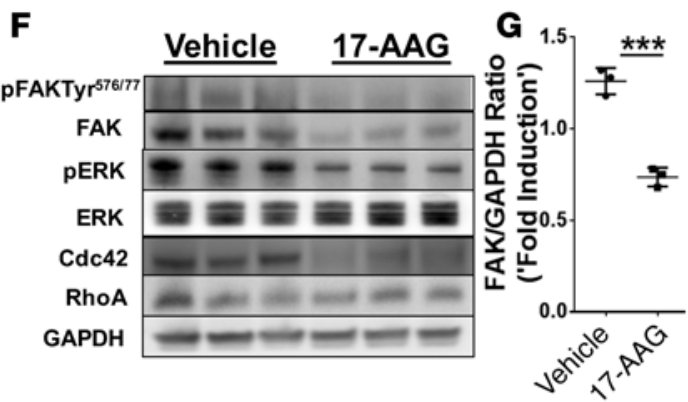

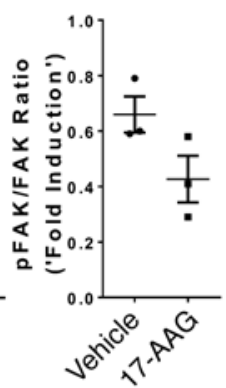

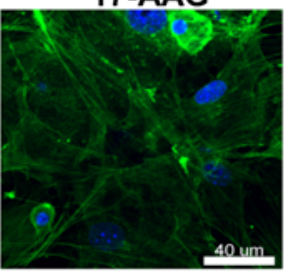

H $\mathrm{Cc/3}$

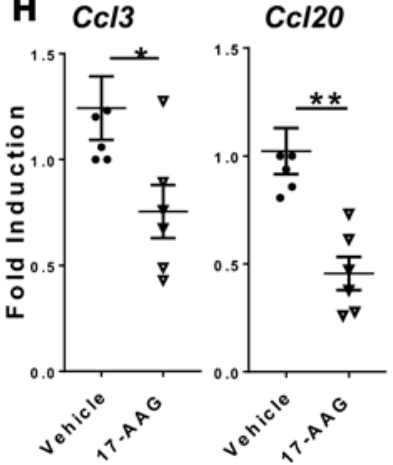

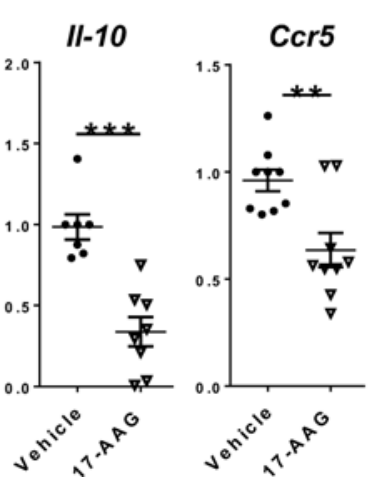

I

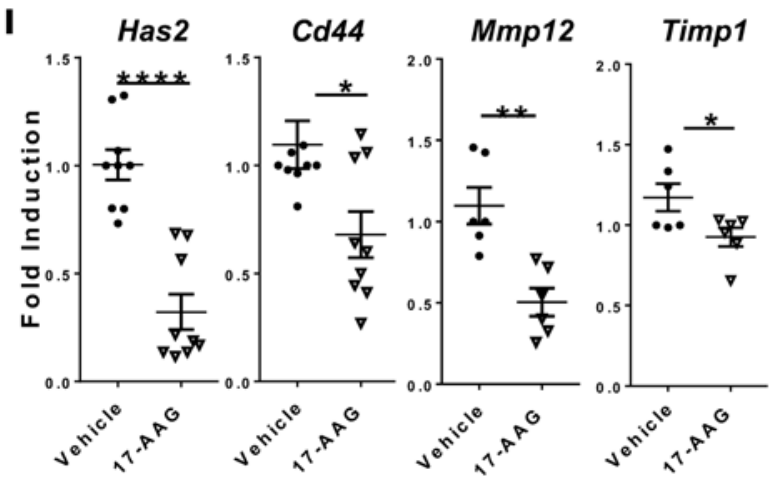

Figure 3. Mechanisms of Hsp90-driven migration and invasion in fibroblasts. Primary lung fibroblasts (CD45-Col1+) isolated from lung fibroblast cultures of human idiopathic pulmonary fibrosis (IPF) or TGF- $\alpha$-transgenic (TGF- $\alpha$-Tg) mice on doxycycline (Dox) for 4 weeks by negative selection with anti-CD45 magnetic beads. (A) Quantification of migration of IPF fibroblasts treated with vehicle or 17-AAG (1 $\mu$ M) for 24 hours ( $n=6 /$ group). (B) Quantification of invasiveness of IPF fibroblasts treated with vehicle or 17-AAC (1 $\mu$ M) for 24 hours ( $n=6 /$ group). (C) Fibroblasts of TCF- $\alpha$-Tg mice on Dox for 4 weeks were transiently transfected with control, Hsp90AA-specific, or Hsp90AB-specific siRNA for 48 hours and the migration was quantified for 24 hours ( $n=6 /$ group). (D) Costaining of F-actin and Hsp90 in lung fibroblasts of TCF- $\alpha$-Tg mice on Dox for 4 weeks. Hsp90 colocalized (white signal) with F-actin in cytoplasmic fibrillary adhesions or focal complexes. Images were obtained at an original magnification of $\times 40$. Scale bars: $40 \mu \mathrm{m}$. (E) F-actin stained using phalloidin in lung fibroblasts of TGF- $\alpha$-Tg mice on Dox for 4 weeks treated with vehicle or 17-AAC (1 $\mu$ M) for 24 hours. Scale bars: $40 \mu \mathrm{m}$. (F) Immunoblot analysis for FAK, p-Tyr ${ }^{576 / 577} F A K$, ERK, p-Tyr ${ }^{468}$ ERK, cell division control protein 42 homolog (Cdc42), and Ras homolog gene family, member A (RhoA) in the lysates of lung fibroblast of TCF- $\alpha$-Tg mice on Dox for 4 weeks treated with vehicle or 17-AAG (0.5 $\mu \mathrm{M})$ for 48 hours. (G) The total expression and phosphorylation of the indicated proteins were measured and normalized to GAPDH levels in the total lysates ( $n=3$ /group). (H) Quantification of Ccl3, CcI20, II-10, and Ccr5 gene transcripts relative to Hprt in the lung fibroblasts of TCF- $\alpha$-Tg mice on Dox for 4 weeks and treated with vehicle or 17-AAG $(0.5 \mu \mathrm{M})$ for 24 hours. (I) Quantification of Has2, Cd44, Mmp12, and Timp1 gene transcripts relative to Hprt in the lung fibroblasts of TGF- $\alpha-T g$ mice on Dox for 4 weeks and treated with vehicle or 17-AAC ( $0.5 \mu M)$ for 24 hours ( $n=3 /$ group). Results are representation of 3 independent experiments with similar results. Data shown are mean \pm SEM. An unpaired 2-tailed Student's $t$ test was performed to measure the significance. ${ }^{*} P<0.05,{ }^{* *} P<0.005,{ }^{* *} P<0.0005$, and ${ }^{* * *} P<0.00005$. 
Inhibition of Hsp90 attenuates fibroblast migration and invasiveness. Excessive migration and invasiveness are hallmarks of fibroblast activation that contribute to severe fibrotic lung disease $(6,7,41)$. To identify whether inhibition of Hsp90 ATPase activity impedes migration and invasiveness of IPF fibroblasts, we performed real-time 3D scratch assays in the presence and absence of 17-AAG. Upon treatment with 17-AAG, resident lung fibroblasts showed a significant reduction in the ability to migrate through Matrigel (invasiveness) (Figure 3, A and B). Similarly, we observed diminished migration and invasion of resident lung fibroblasts treated with 17-AAG compared with vehicle-treated resident lung fibroblasts isolated from the fibrotic lungs of TGF- $\alpha-$ Tg mice on Dox for 4 weeks (Supplemental Figure 1A). Fibrocytes have been shown to migrate and invade into the fibrotic lesions to augment the progression of fibrosis in mouse models of pulmonary fibrosis $(6,15)$. In IPF, the number of fibrocytes in lung biopsies and circulating blood has been shown to associate with severity of fibrotic lung disease $(42,43)$. Therefore, we assessed whether 17-AAG inhibits the motility of fibrocytes isolated from human IPF lungs and also fibrotic lungs of TGF- $\alpha-\mathrm{Tg}$ mice on Dox for 4 weeks. Inhibition of Hsp90 ATPase activity resulted in a marked decrease in the migration and invasion of fibrocytes isolated from both IPF lungs (Supplemental Figure 1B) and lungs of TGF- $\alpha-\mathrm{Tg}$ mice (data not shown). In mammalian cells, Hsp90AA and Hsp90AB are the 2 major isoforms involved in Hsp90-driven functions (44). Although both isoforms bind similarly to their intracellular cochaperones and display ATPase activity, in some cases Hsp90AA and Hsp90AB have differential effects on substrate interactions $(45,46)$. To assess the relative contributions of Hsp90 isoforms on fibroblast migration, we measured migration of lung fibroblasts that were treated with siRNA specific for either Hsp90AA or Hsp90AB mRNA, as well as control siRNA. Treatment of fibroblasts with Hsp90AA or Hsp90AB siRNA specifically knocked down the corresponding isoform expression compared with control siRNA (Supplemental Figure 2, A and B). Fibroblast migration was significantly attenuated with the deficiency of either Hsp90AA or Hsp90AB isoform compared with control siRNA treatment (Figure 3C). Together, these data demonstrate that the migration capacity of fibroblasts was regulated by both Hsp90AA and $\mathrm{Hsp} 90 \mathrm{AB}$ isoforms.

To investigate the mechanisms of Hsp90-driven migration and invasion, lung fibroblasts were costained for F-actin and Hsp90. Notably, we observed a strong colocalization of cytoplasmic Hsp90 with F-actin filaments in fibroblasts (Figure 3D). Further, the formation of F-actin filaments was attenuated in fibroblasts treated with 17-AAG compared with vehicle treatment (Figure 3E). To determine whether the formation of F-actin filaments by Hsp90 was due to altered signaling via pathways involved in F-actin formation, we assessed focal adhesion kinase (FAK), CDC42, and phosphorylation of ERK. Inhibition of Hsp90 ATPase activity resulted in a significant decrease in the protein levels of total FAK and CDC42, and also phosphorylation of ERK, but had no effect on the phospho-FAK/FAK ratio, total ERK, and RhoA protein levels (Figure 3, F and G).

Both migration and invasion of fibroblasts are regulated by altered expression of chemokines, cytokines, and proteases in the lungs of $\operatorname{IPF}(1,7,47,48)$. To address whether Hsp90 inhibition alters the expression of genes involved fibroblast invasiveness, resident lung fibroblasts were treated with 17-AAG for 24 hours and transcripts were analyzed using quantitative reverse transcription PCR (qRT-PCR). Treatment with 17-AAG resulted in a significant decrease in the levels of chemokines and cytokines ( $\mathrm{Ccl} 3$, Ccl20, Ccr5, and I1-10) (Figure $3 \mathrm{H}$ ), as well as genes involved in the invasiveness of fibroblasts (Has2, Cd44, Mmp12, and Timp1) (Figure 3I), while treatment with 17-AAG had no effect on expression of Mmp2, Mmp9, or Timp2 (Supplemental Figure 3). Thus, the above data suggest that Hsp90 induces signaling and the expression of genes involved in migration and invasiveness of fibroblasts in IPF.

Inhibition of Hsp90 attenuates fibroproliferation. The proliferative expansion of resident lung fibroblasts at the site of injury is a critical pathological process during initiation and maintenance of fibrotic lesions in the lung $(6,15)$. To identify the effect of Hsp90 inhibition on fibroproliferation, we treated lung fibroblasts of human IPF with 17-AAG or vehicle for 24 hours. Inhibition of Hsp90 ATPase activity resulted in a significant decrease in the percentage of proliferating cell nuclear antigen-positive (PCNA-positive) fibroblasts compared with vehicle treatment (Figure 4, A and B). Similarly, the proliferation of lung fibroblasts isolated from the fibrotic lungs of TGF- $\alpha-\mathrm{Tg}$ mice was inhibited in a dose-dependent manner, as the concentration of 17-AAG was increased from $0.01 \mu \mathrm{M}$ to $1 \mu \mathrm{M}$ at 48 hours (Figure $4 \mathrm{C}$ ). To evaluate the role of Hsp90 isoforms in proliferation, lung fibroblasts were treated with siRNA specific for either Hsp90AA or Hsp90AB mRNA, as well as control siRNA for 72 hours and BrdU incorporation was assessed to quantify the fibroblast proliferation. The loss of Hsp90AB was sufficient to inhibit proliferation of fibroblasts compared with Hsp90AA siRNA-treated or control siRNA-treated cells (Figure 4D). To assess the role of 
A

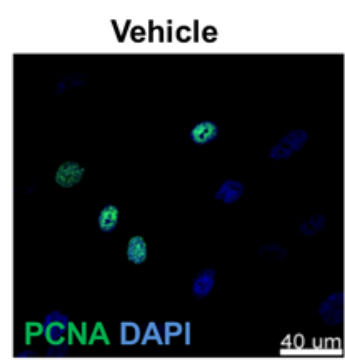

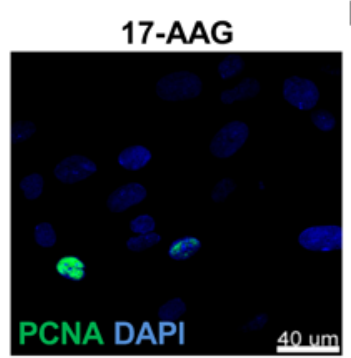

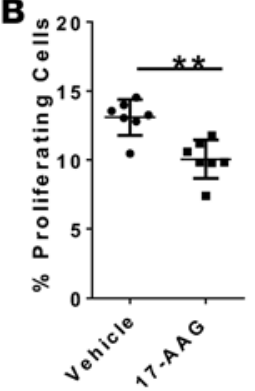

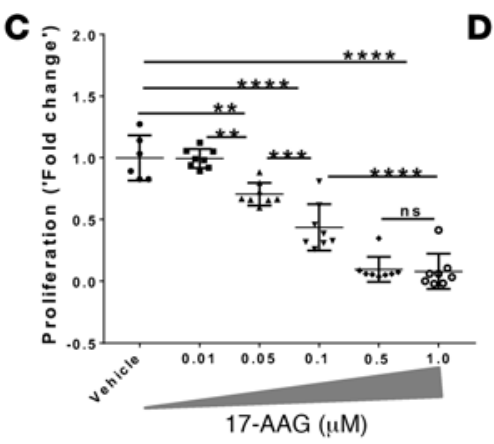

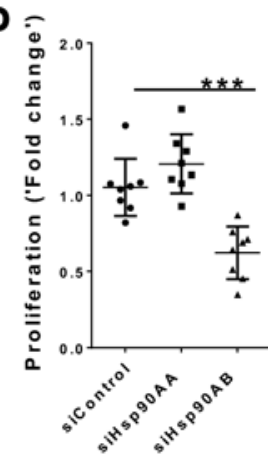

E

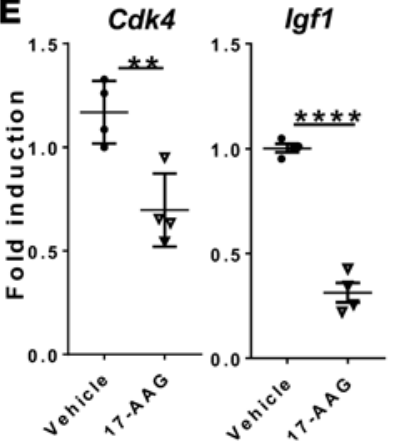

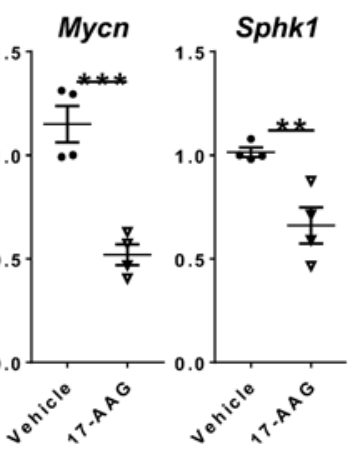
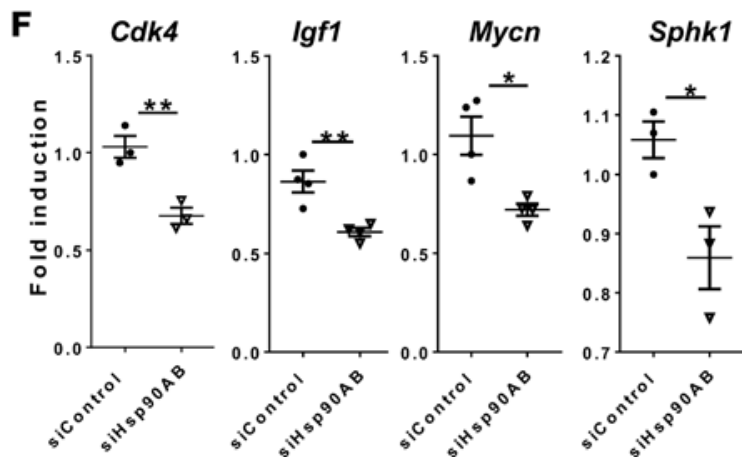

Figure 4. Hsp90 regulates the proliferation of fibroblasts in fibrotic lesions. (A) Primary lung fibroblasts (CD45-Col1+) isolated from lung fibroblast cultures of human idiopathic pulmonary fibrosis (IPF) lungs by negative selection with anti-CD45 magnetic beads. Fibroblasts were treated with vehicle or 17-AAG $(0.5 \mu \mathrm{M})$ for 24 hours and immunostained with antibodies against proliferating cell nuclear antigen (PCNA). Scale bars: $40 \mu \mathrm{m}$. (B) The number of PCNA-positive cells and total DAPI-positive cells were quantified using MetaMorph image analysis software ( $n=3-4 /$ group). Proliferation is indicated as the percentage of proliferating cells in total DAPI-positive cells. Results are cumulative of 2 independent experiments ( $n=3-4 / g r o u p)$. (C) Quantification of proliferation using the BrdU incorporation assay in lung resident fibroblasts isolated from lung cell cultures of TCF- $\alpha$-transgenic (TCF- $\alpha$-Tg) mice on doxycycline (Dox) for 4 weeks. Fibroblasts treated with indicated doses of 17-AAC for 48 hours from beginning of the treatment relative to vehicle is plotted. One-way ANOVA with Sidak's multiple comparisons test was used to measure significant difference. (D) Extent of proliferation was measured using BrdU incorporation assay in lung fibroblasts isolated from lung cell cultures of TCF- $\alpha-T g$ mice on Dox for 4 weeks and treated with control, Hsp90AAspecific, or Hsp90AB-specific siRNA for 72 hours. Results are cumulative of 2 independent experiments ( $n=4 / g r o u p$ ). One-way ANOVA with Sidak's multiple comparisons test was used to measure significant difference. (E) Quantification of Cdk4, Igf1, Mycn, and Sphk1 gene transcripts relative to Hprt in the lung-resident fibroblasts of TGF- $\alpha-T g$ mice on Dox for 4 weeks and treated with vehicle or 17-AAG (0.2 $\mu$ M) for 24 hours. Results are representative of 2 independent experiments with similar results ( $n=4$ /group). (F) Quantification of Cdk4, Igf1, Mycn, and Sphk1 gene transcripts relative to Hprt in the lung fibroblasts of TCF- $\alpha$-Tg mice on Dox for 4 weeks and treated with control, Hsp90AA-specific, or Hsp90AB-specific siRNA for 72 hours. Results are representative of 2 independent experiments ( $n=3-4$ /group). Data shown are mean \pm SEM. An unpaired 2 -tailed Student's $t$ test was performed to measure the significance. ${ }^{*} P<0.05,{ }^{* *} P<0.005,{ }^{* *} P<0.0005$, and ${ }^{* * *} P<0.00005$.

Hsp90 in fibroproliferation, primary fibroblasts were treated with either 17-AAG or Hsp90AB siRNA, and Hsp90-driven IPF network genes predicted to regulate fibroproliferation, such as Cdk4, Igf1, Mycn, and Sphk1, were quantified (Figure 1A). Notably, either pharmacological inhibition of Hsp90 ATPase activity with 17-AAG or genetic knockdown of Hsp90AB was sufficient to attenuate the expression of Hsp90driven genes involved in fibroproliferation (Figure 4, E and F). In contrast, Hsp90AA siRNA treatment had no effect on proliferation or genes involved in fibroproliferation (Figure 4D and Supplemental Figure 4). These findings clearly establish that $\mathrm{Hsp} 90 \mathrm{AB}$ and its ATPase activity may function as positive regulators of fibroproliferation in IPF.

Inhibition of Hsp90 attenuates ECM production. Myofibroblasts are the major effector cells responsible for the excessive ECM deposition in the fibrotic lesions of human IPF (49-51). To investigate whether inhibition of Hsp90 downregulates $\alpha$-smooth muscle actin ( $\alpha$ Sma) and ECM gene expression, lung fibroblasts were treated with 17-AAG or vehicle for 24 hours and transcript levels of $\alpha$ Sma, fibronectin (Fn1), collagen type $1 \alpha(\mathrm{Co} 11 \alpha)$, and Col5 $\alpha$ were quantified by qRT-PCR. Inhibition of Hsp90 ATPase activity resulted in a significant downregulation of ECM genes (Figure 5A). To determine the role of Hsp90AA and Hsp90AB in ECM production, lung fibroblasts were treated with Hsp90AA- or Hsp90AB-specific siRNA or control siRNA for 72 hours. Notably, loss of Hsp90AB was sufficient to attenuate the expression of ECM genes, such as $\alpha \mathrm{Sma}$, Col1 $\alpha$, and Col5 $\alpha$, but had no effect on Fn1 compared with control siRNA (Figure 5B). Hsp90AA siRNA treatment had no effect on the ECM genes (Supplemental Figure 5). 

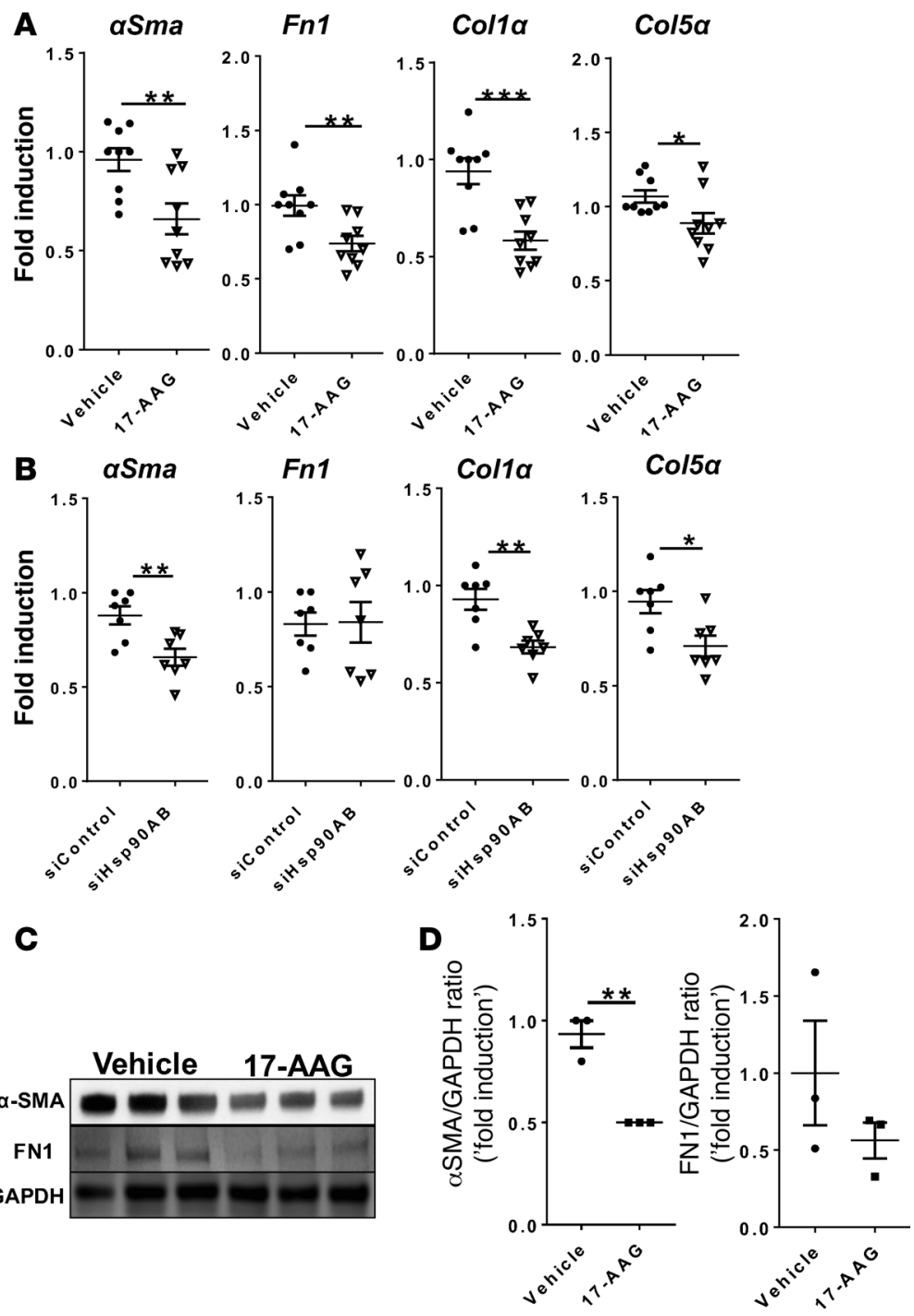

Figure 5. Hsp90 regulates $\alpha$ SMA and extracellular matrix gene expression. Primary lung fibroblasts ( $\left.\mathrm{CD} 45^{-} \mathrm{Co} 11^{+}\right)$isolated from lung fibroblast cultures of TGF- $\alpha$-transgenic mice on doxycycline (Dox) for 4 weeks and treated with vehicle or 17-AAG and control, Hsp90AA-specific, or Hsp90AB-specific siRNA. (A) Quantification of $\alpha \mathrm{Sma}$, Fn1, Col1 $\alpha$ and Col $5 \alpha$ gene transcripts relative to $\mathrm{Hprt}$ in the lung-resident fibroblasts treated with vehicle or 17-AAG $(1 \mu \mathrm{M})$ for 24 hours. Data are cumulative of 3 independent experiments with similar findings ( $n=3$ /group). (B) Quantification of $\alpha \mathrm{Sma}$, Fn1, Col1 $\alpha$, and Col5 $\alpha$ gene transcripts relative to $\mathrm{Hprt}$ in the lung-resident fibroblasts treated with control, Hsp90AA-specific, or Hsp90AB-specific siRNA for 72 hours. Data are cumulative of 2 independent experiments with similar findings ( $n=3-4$ / group). (C) Immunoblots for $\alpha$ SMA and FN1 in the lysates of lung fibroblasts treated with vehicle or 17-AAG $(1 \mu \mathrm{M})$ for 48 hours. (D) Quantification of $\alpha S M A$ and FN1 proteins normalized to GAPDH levels in the total lysates of lung fibroblasts treated with vehicle or 17-AAG $(1 \mu \mathrm{M})$ for 24 hours. Data are representative of 2 independent experiments with similar findings ( $n=3$ /group). Data shown are mean \pm SEM. An unpaired 2-tailed Student's $t$ test was performed to measure the significance. ${ }^{*} P<0.05,{ }^{*} P<0.005$, and ${ }^{* * *} P<0.0005$.

Furthermore, Western blot analysis demonstrated that 17-AAG inhibits protein levels of $\alpha \mathrm{SMA}$ and FN1 in fibroblasts isolated from the fibrotic lungs of TGF- $\alpha-$ Tg mice on Dox for 4 weeks (Figure 5, C and D).

TGF- $\beta$ is a master regulator of fibroblast to myofibroblast transformation and expression of ECM genes in pulmonary fibrosis $(52,53)$. To determine whether inhibition of Hsp90 ATPase activity is sufficient to attenuate TGF- $\beta$-induced myofibroblast transformation, we performed lineage tracing studies using lung resident fibroblasts isolated from $\alpha$ SMA reporter mice $\left(\alpha S M A^{\text {CreER }}{ }^{\text {ROSA }}{ }^{\mathrm{mTmG}}\right.$ mice). Primary fibroblasts were cultured in the presence of tamoxifen and 17-AAG or vehicle and treated with TGF- $\beta$ for 72 hours to visualize and quantify transformation of fibroblast to myofi-

broblasts. In this system, upon activation of $\alpha \mathrm{SMA}$, fibroblasts change expression of membrane tomato (red; $\mathrm{mT}$ ) to membrane GFP (green; $\mathrm{mG}$ ). As anticipated, we observed a significant increase in $\alpha$ SMAexpressing cells (green) by treatment with TGF- $\beta$, and treatment with 17-AAG was sufficient to attenuate the number of $\mathrm{mG}$-expressing cells (Figure 6, A and B). To determine whether 17-AAG attenuates TGF- $\beta$-induced ECM gene expression, human fibroblasts were treated with TGF- $\beta$ in the presence and absence of 17-AAG and transcripts of ECM genes were quantified by qRT-PCR. As predicted, TGF- $\beta$ induced the expression of COL1 $\alpha$ and FN1 (Figure 6, C and D). Notably, treatment with 17-AAG was sufficient to attenuate TGF- $\beta$-driven expression of COL1 $\alpha$ and FN1. As we observed differential regulation of proliferation and ECM production, we investigated if $\mathrm{Hsp} 90$ isoforms play specific or redundant functional roles in the transformation of fibroblasts to myofibroblasts. Notably, deficiency of Hsp90AB but not Hsp90AA significantly attenuated TGF- $\beta$-driven fibroblast to myofibroblast transformation compared with control siRNA treatment (Figure 6E). Together, our in vitro results establish that either inhibition of Hsp90 ATPase activity or Hsp90AB expression was sufficient to attenuate TGF- $\beta$-driven myofibroblast transformation and ECM gene expression.

Inhibition of Hsp90 ATPase activity attenuates IPF fibroblast activation. To identify whether inhibition of Hsp90 ATPase activity regulates profibrotic processes of IPF fibroblasts, we treated human IPF fibroblasts with vehicle or 17-AAG and assessed signaling pathways involved in F-actin formation and expression of genes involved in proliferation, migration, invasion and ECM deposition. Treatment with 17-AAG resulted in a significant decrease in the protein levels of total FAK but had no effect on the phospho-FAK/FAK ratio, 

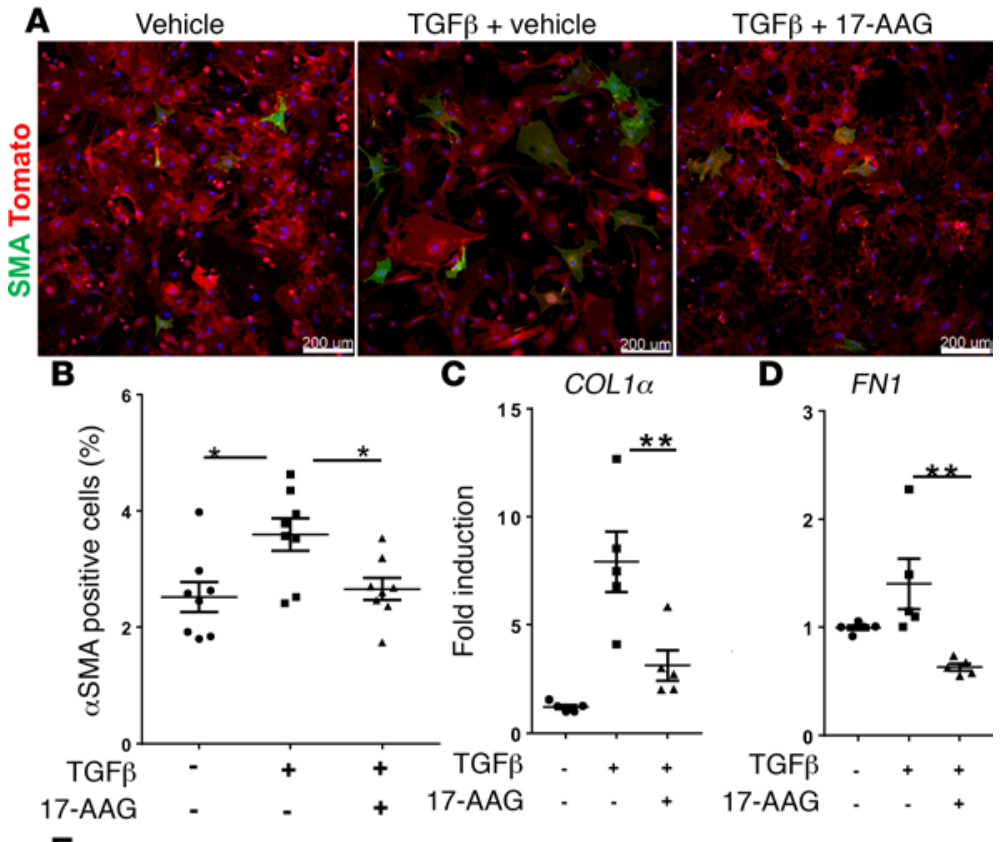

E

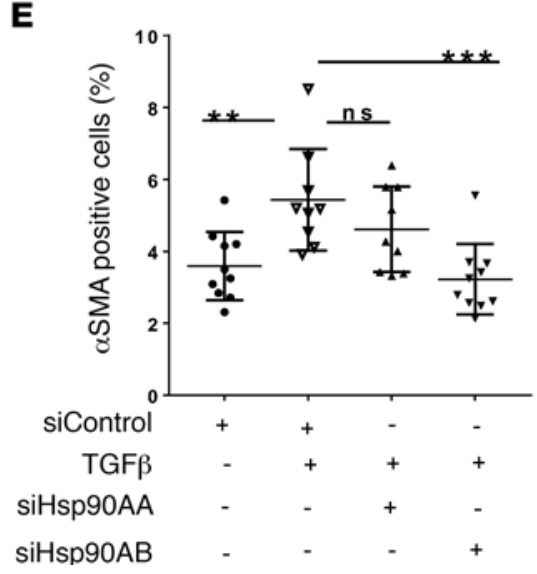

Figure 6. Hsp90 inhibition attenuates TGF- $\beta$-induced myofibroblast transformation and ECM gene expression. (A) Primary lung-resident fibroblasts $\left(\mathrm{CD}_{4} 5^{-} \mathrm{Col} 1^{+}\right)$were isolated from lung cell cultures of $\alpha S M A^{\text {creER }} R O S A^{\mathrm{mTmG}}$ mice and treated with TCF- $\beta$ and 4-hydroxy tamoxifen in the presence and absence of 17-AAG for 72 hours. Immunofluorescence images were obtained at an original magnification of $\times 10$. Scale bars: $200 \mu \mathrm{m}$. (B) The number of GFP-positive and total DAPI-positive (blue) cells were quantified using MetaMorph image analysis software ( $n=4-5$ / group) and are indicated as the percentage $\alpha$ SMA-positive cells in total DAPI-positive cells. Data are cumulative of 2 independent experiments with similar results. (C and D) Human lungresident fibroblasts (CD45-Col1+) were isolated from non-idiopathic pulmonary fibrosis (non-IPF) lung fibroblast cultures and treated with TCF- $\beta$ in the presence and absence of 17-AAG for 24 hours. Transcripts of extracellular matrix (ECM) genes COL1 $\alpha$ and FN1 were quantified using QRT-PCR and are shown as the fold-induced gene transcripts relative to GAPDH. Data are representative of 2 independent experiments with similar results $(n=$ 4-5/group). (E) Lung-resident fibroblasts of $\alpha S M A^{\text {creer }}{ }^{\text {ROSA }} A^{\text {MTmG }}$ mice were transfected with control, Hsp90AA-specific, or Hsp90AB-specific siRNA. After a 24-hour transfection, cells were treated with TCF- $\beta$ and 4-hydroxy tamoxifen for 72 hours and the number of GFP-positive and total DAPI-positive cells were quantified using MetaMorph image analysis software ( $n=$ 4-5/group). Data are cumulative of 2 independent experiments with similar results. One-way ANOVA with Sidak's multiple comparisons test was used to measure significant difference for all the experiments. All data shown are mean $\pm \mathrm{SEM}$. ${ }^{*} P<0.05$, ${ }^{* *} P<0.005$, and ${ }^{* *} P<0.0005$. ns, not significant.

total ERK, CDC42, and RhoA protein levels (Figure 7, A and B). Inhibition of Hsp90 ATPase activity resulted in a significant reduction in the expression of genes involved in migration and invasiveness of IPF fibroblasts (Figure 7C). Similarly, pharmacological inhibition of Hsp90 ATPase activity with 17-AAG was sufficient to attenuate the expression of genes involved in fibroproliferation (CDK4, IGF1, and SPHK1) and ECM deposition (COL1 $\alpha$, COL $5 \alpha$, and

FN1) (Figure 7, D and E). Thus, the ATPase activity of Hsp90 induces proliferation, migration, invasion, and ECM deposition in IPF fibroblasts, which was also seen in the fibroblasts of the TGF- $\alpha$ model.

Inhibition of Hsp90 ATPase activity attenuates pulmonary fibrosis in vivo. We evaluated the potential benefits of Hsp90 inhibition in pulmonary fibrosis using a mouse model of TGF- $\alpha$-induced pulmonary fibrosis. Our previous studies have demonstrated that conditional overexpression of TGF- $\alpha$ induces progressive fibrosis in the adventitia, parenchyma, and subpleural areas of the lung with histologic features and genomic profiles similar to human IPF $(16,54,55)$. At 3 weeks on Dox, TGF- $\alpha-$ Tg mice developed detectable fibrosis in subpleural and adventitial areas of the lung and also modest increases in lung weights and hydroxyproline levels (Supplemental Figure 6). The above changes progressed and increased significantly in TGF- $\alpha-T g$ mice continued on Dox for an additional 3 weeks compared with control mice or mice on Dox for 3 weeks (Supplemental Figure 6). To determine whether Hsp90 inhibition influences the progression of established fibrosis, following 3 weeks of Dox treatment when fibrosis is already manifest, TGF- $\alpha-\mathrm{Tg}$ mice were administered 17-AAG while remaining on Dox for an additional 3 weeks (6 weeks total) (Figure 8A). The right lung weights were increased in TGF- $\alpha-$ Tg mice compared with control mice on Dox for 6 weeks. This increase in lung weights was attenuated in TGF- $\alpha-\mathrm{Tg}$ mice treated with 17-AAG when compared with vehicle-treated TGF- $\alpha-\mathrm{Tg}$ mice (Figure $8 \mathrm{~B}$ ). Similarly, the total lung hydroxyproline levels were increased in TGF- $\alpha-\mathrm{Tg}$ mice treated with either vehicle or 17-AAG (Figure $8 \mathrm{C}$ ). However, this increase in the lung hydroxyproline levels was attenuated in TGF- $\alpha-\mathrm{Tg}$ mice treated with 17-AAG compared with vehicle-treated TGF- $\alpha-$ Tg mice (Figure 8 C). Masson's trichrome staining of lung sections revealed extensive subpleural thickening and adventitial lung fibrosis in TGF- $\alpha-\mathrm{Tg}$ mice compared with vehicle-treated control mice 


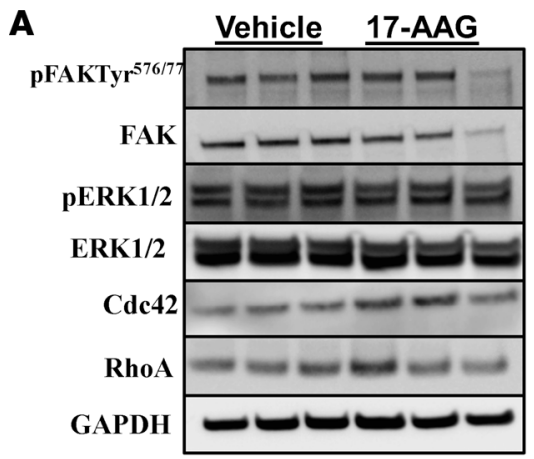

B
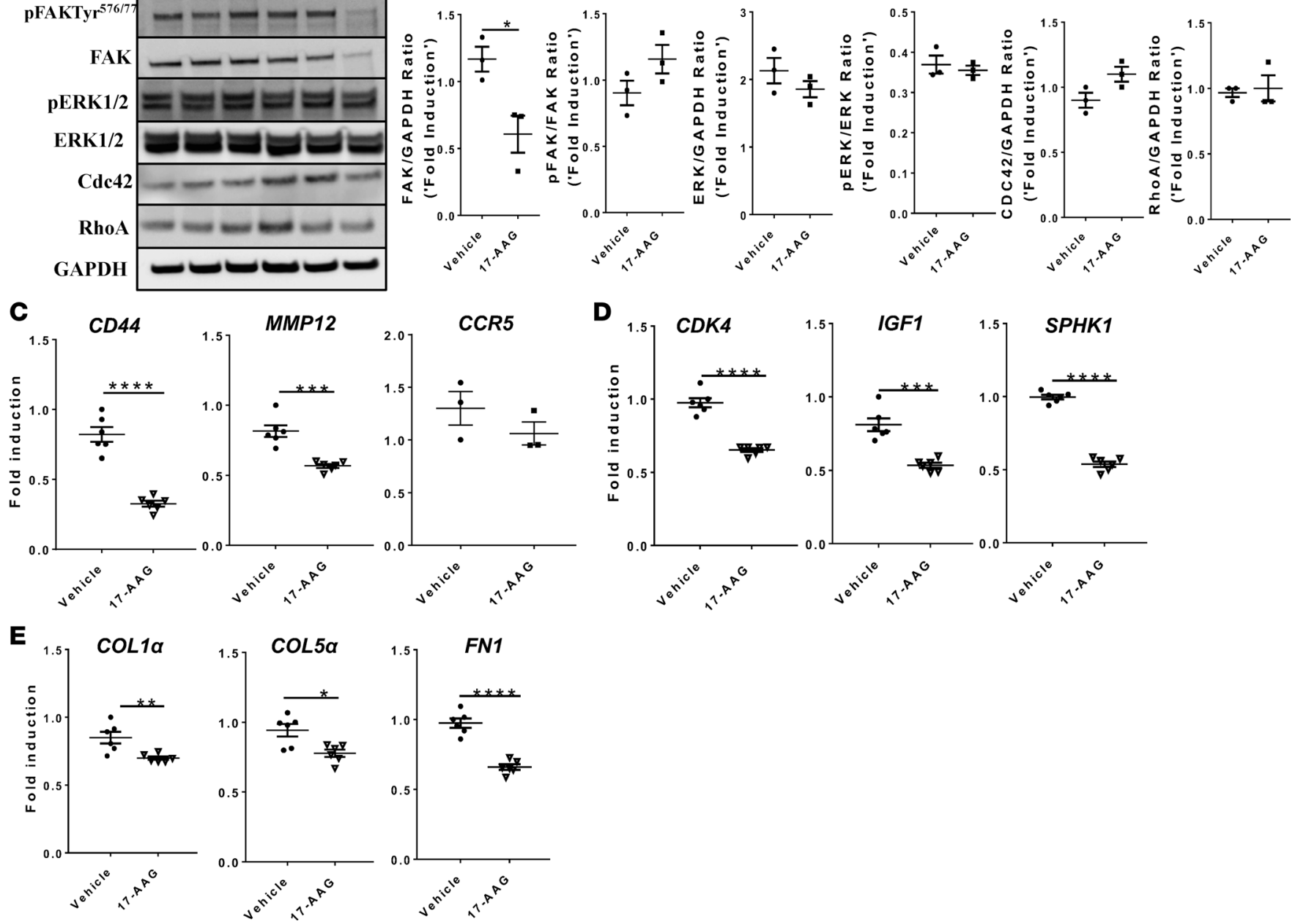

Figure 7. Hsp90 regulates activation of IPF fibroblasts. Primary lung resident fibroblasts ( $\mathrm{CD}^{-} 5^{-}$Col1 ${ }^{+}$) were isolated from human idiopathic pulmonary fibrosis (IPF) lung cell cultures and treated with vehicle or 17-AAG. (A) Immunoblot analysis for FAK, p-Tyr ${ }^{57 / 577 F A K, ~ E R K, ~ p-T y r ~}{ }^{468}$ ERK, CDC42, and RhoA in the lysates of IPF lung fibroblasts treated with vehicle or 17-AAC $(0.5 \mu \mathrm{M})$ for 48 hours. (B) The total expression and phosphorylation of the indicated proteins were measured and normalized to GAPDH levels in the total lysates of IPF lung fibroblasts treated with vehicle or 17-AAG (0.5 $\mu$ M) for 48 hours ( $n$ = 3/group). (C) Quantification of CD44, MMP12, and CCR5 gene transcripts relative to GAPDH in IPF lung fibroblasts treated with vehicle or 17-AAG (0.2 $\mu$ M) for 24 hours ( $n=3-6 /$ group). (D) Quantification of CDK4, IGF1, and SPHK1 gene transcripts relative to GAPDH in IPF lung fibroblasts treated with vehicle or 17-AAG $(0.2 \mu \mathrm{M})$ for 24 hours ( $n=6 /$ group). (E) Quantification of COL $1 \alpha, C O L 5 \alpha$, and FN1 gene transcripts relative to GAPDH in IPF lung fibroblasts treated with vehicle or 17-AAC $(0.5 \mu \mathrm{M})$ for 24 hours ( $n=6$ /group). Data are representative of 2 independent experiments with similar findings. Data shown are mean \pm SEM values. ${ }^{*} P<0.05,{ }^{* *} P<0.005$, ${ }^{* *} P<0.0005$, and ${ }^{* * *} P<0.00005$.

(Figure 8D). However, the therapy with 17-AAG resulted in a significant decrease in subpleural thickening, as well as reduced adventitial fibrosis compared with vehicle-treated TGF- $\alpha-T g$ mice. Increased $\alpha$ SMA and Ki67 immunostaining were detected in the subpleural fibrotic lesions in TGF- $\alpha-\mathrm{Tg}$ mice compared with vehicle-treated control mice, which was significantly diminished by $17-\mathrm{AAG}$ treatment (Figure 8, E and F). Further, we quantified the changes in fibrosis specifically in the subpleural region by measuring the pleural thickness in the lung sections from all groups. Not surprisingly, TGF- $\alpha-$ Tg mice show a significant increase in subpleural thickness after 6 weeks on Dox treatment compared with nontransgenic control mice. This increase in subpleural thickness was attenuated in TGF- $\alpha-\mathrm{Tg}$ mice administered 17-AAG compared with vehicle-treated TGF- $\alpha-\mathrm{Tg}$ mice (Figure $8 \mathrm{G}$ ). We evaluated the mean alveolar chord length to determine the potential toxic effects of Hsp90 inhibition on lung alveolar integrity during TGF- $\alpha$-induced pulmonary fibrosis. The mean alveolar chord length was increased in Dox-induced TGF- $\alpha-\mathrm{Tg}$ mice compared with control mice treated with Dox for 6 weeks (Figure 8H). This increase in alveolar chord length was not altered in TGF- $\alpha-\mathrm{Tg}$ mice treated with 17-AAG when compared with vehicle-treated TGF- $\alpha-\mathrm{Tg}$ mice. Thus, our in vivo data suggest that Hsp90 plays a critical role in pulmonary fibrosis and inhibition of 
A
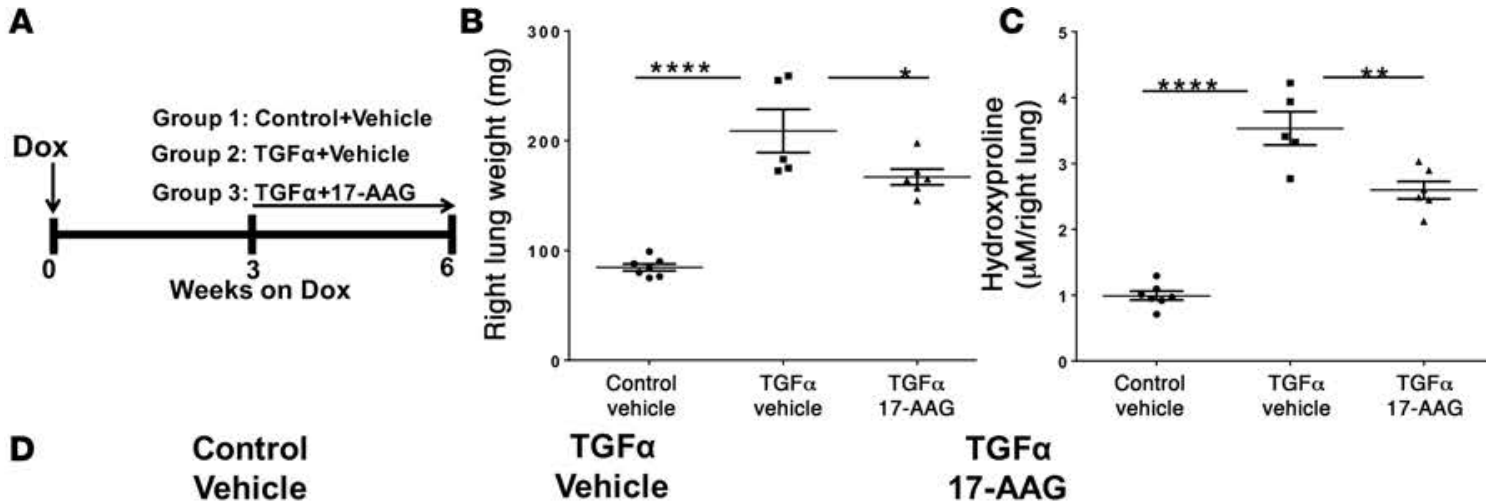

TGFa

TGFa

Vehicle

17-AAG

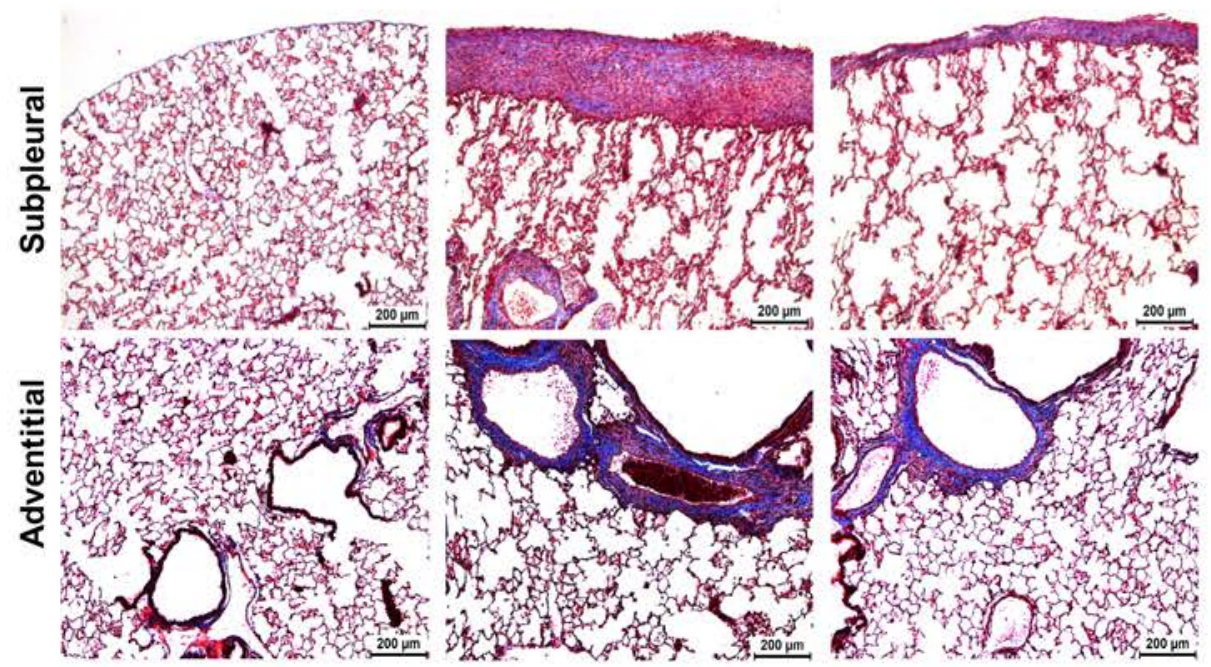

E

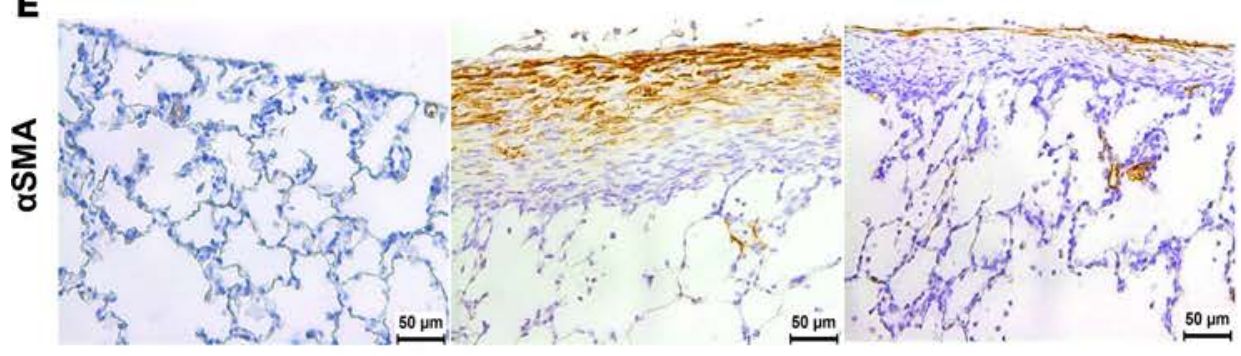

$\mathbf{F}$

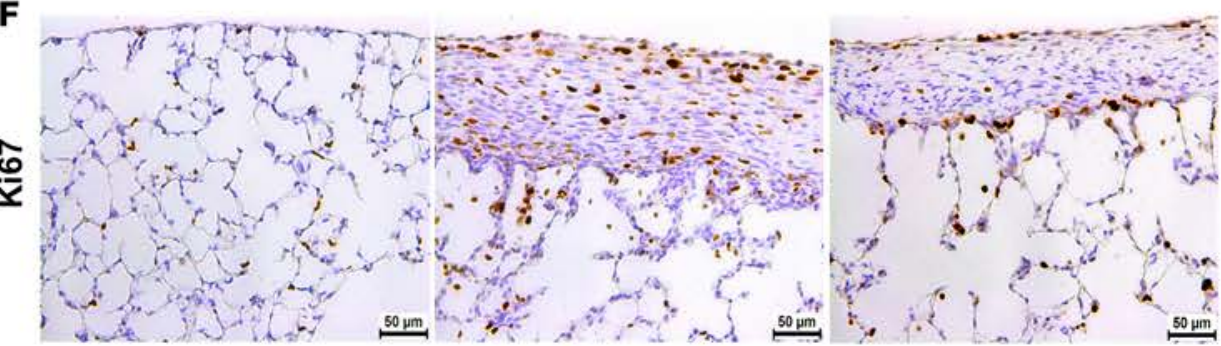

G

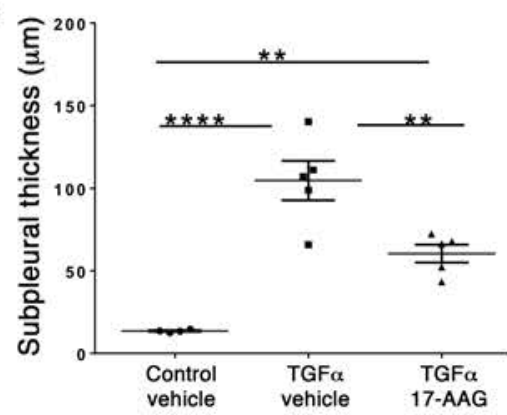

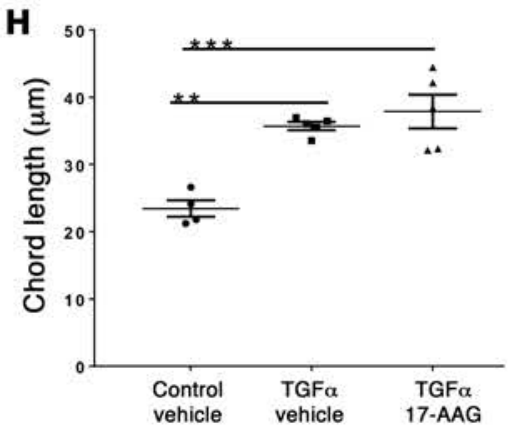

Figure 8. Therapeutic administration of 17-AAG attenuates pulmonary fibrosis in vivo. (A) Schematic representation of 17-AAC treatment protocol in vivo. Control and TGF- $\alpha$-transgenic (TGF- $\alpha-\mathrm{Tg}$ ) mice were treated with vehicle or 17-AAC ( $15 \mathrm{mg} / \mathrm{kg}$; once per day) for the last 3 weeks while remaining on doxycycline (Dox) for a total of 6 weeks. (B) Quantification of the right-lung weight of mice treated with vehicle or 17-AAG ( $n=5-7)$ group). (C) Quantification of total lung hydroxyproline levels in mice treated with vehicle or 17-AAC ( $n=5-7 /$ group). (D) Images of Masson's trichromestained lung sections from all the groups. Top, subpleural regions of the lung; bottom, adventitia. All images were taken at an original magnification of $\times 10$. Scale bars: $200 \mu \mathrm{m}$. Images are representative of each group ( $n=5-7 /$ group). (E) Assessment of aSMA immunoreactivity by immunohistochemistry in the subpleural fibrotic lesions of the lung sections from each group ( $n=5 /$ group). All images were taken at an original magnification of $\times 20$. Scale bars: $50 \mu \mathrm{m}$. (F) Assessment of Ki67 immunoreactivity in the subpleural fibrotic lesions of the lung sections from each group ( $n=5$ /group). All images were taken at an original magnification of $\times 20$. Scale bars: $50 \mu \mathrm{m}$. (C) Subpleural thickness was quantified in the Masson's trichrome-stained lung sections of control mice and TGF- $\alpha-T g$ mice treated with vehicle or 17-AAG ( $n=4-5$ /group). (G) Mean chord length was quantified in the Masson's trichrome-stained lung sections of control mice and TCF- $\alpha-\mathrm{Tg}$ mice treated with vehicle or 17-AAG ( $n$ $=4-5$ (group). Statistical significance was measured using 1-way ANOVA with Sidak's multiple comparison test for all the experiments. ${ }^{*} P<0.05,{ }^{* *} P<0.005$, ${ }^{* * *} P<0.0005$, and ${ }^{* * *} P<0.00005$. 
Hsp90 using 17-AAG attenuates TGF- $\alpha$-induced progressive subpleural thickening and pulmonary fibrosis. Together, these studies demonstrate that Hsp90 inhibition at the time of extensive and established fibrosis modulates the progression of the disease based on biochemical and histologic parameters.

\section{Discussion}

The lack of knowledge regarding the molecular regulators that initiate and maintain key fibrogenic processes is the major limitation in developing effective new therapies for IPF. Here, we adopted an unbiased chemical genomic approach and identified $\mathrm{Hsp} 90$ as a potentially novel regulator of fibroblast activation in the pathogenesis of IPF. Hsp90 was ranked as one of the top therapeutic drug candidates with antifibrotic and therapeutic potential based on reversing IPF gene signatures involved in fibroblast activation, such as proliferation, growth, motility, and ECM production. Our findings offer a preclinical validation of Hsp90 as a potential therapeutic candidate for fibrotic lung disease and also mechanistic details for Hsp90-driven fibroblast activation. We identified Hsp90-driven gene networks that were fibroblast-specific and previously associated with IPF, such as genes involved in dysregulated tissue remodeling (COL1 $\alpha$, COL5 $\alpha$, COL6 $\alpha$, aSMA, and MMPs), fibroproliferation (IGF1, SPHK1, CDK4, and MYCN), and fibroblast motility (CCL2, CCL3, CCL20, IL-6,IL-10, HAS2, CD44, and CCR5). One of the most consistently upregulated gene axes in IPF was HAS2 and CD44 transcripts, which have been shown to regulate invasiveness of fibroblasts in severe fibrotic lung disease $(6,7,41)$. Consistent with genomics data, we observed a strong immunoreactivity against Hsp90 protein in the lung sections of human IPF that predominantly contains the mesenchymal component. Hsp90 ATPase activity was found to be elevated in fibroblasts isolated from lungs with active fibrosis, in both IPF lungs and the TGF- $\alpha$ mouse model of pulmonary fibrosis. Further, Hsp90 exhibited a higher affinity for 17-AAG than its parent compound, geldanamycin, in fibrotic protein lysates compared with nonfibrotic controls. The findings suggest that 17-AAG can selectively target Hsp90 of fibrotic lungs due to its higher binding affinity.

Hsp90 is a molecule of emerging interest in studies of oncology and several chronic tissue remodeling diseases of the lung, heart, liver, joints, and kidney $(30,31,35,56)$. Our findings demonstrate that pharmacological inhibition of Hsp90 attenuated fibroproliferation, ECM production, and myofibroblast accumulation, both in vitro and in vivo. In particular, chronic administration of a pharmacologically relevant dose of $15 \mathrm{mg} / \mathrm{kg}$ 17-AAG over 3 weeks resulted in significant reduction in subpleural thickness, collagen deposition, and ECM-producing myofibroblasts in a mouse model of TGF- $\alpha$-induced pulmonary fibrosis. Pulmonary fibrosis in the TGF- $\alpha$-overexpressing mouse model resembles clinical features observed in human disease, including the excessive proliferation of fibroblasts, ECM deposition, and myofibroblast transformation, as well as progressive subpleural thickening radiating toward the parenchyma $(15,16,57)$. We did not observe toxicities from 17-AAG in this study, but higher doses of 17-AAG than those used here have been shown to induce toxicity in mice (58-60). In fact, our immunostaining suggests that airway and alveolar epithelial cells also expressed significant amounts of Hsp90, which might play a role in epithelial cell functions in IPF. Therefore, future studies are warranted to identify and validate the client proteins of Hsp90 that regulate myofibroblasts and other lung cell functions in IPF.

The role of TGF- $\beta$ has been well established in fibrotic diseases, where overexpression of TGF- $\beta$ alone induces fibrosis in skin, kidney, heart, and lung (61-63). TGF- $\beta$ signals through heteromeric complexes to activate Smad2 and Smad3 (Smad2/3), which then forms a heteromeric complex with Smad4 that regulates transcription of genes involved in fibroblast activation, differentiation, and ECM production. Moreover, TGF- $\beta$ has been shown to play an important role in the maintenance of established fibrosis in a TGF- $\alpha$-driven pulmonary fibrosis mouse model (64). Our data demonstrate that supplementation of TGF- $\beta$ alone was sufficient to induce fibroblast to myofibroblast transformation and ECM gene expression in fibroblast cultures, and that the addition of 17-AAG or the loss of the Hsp90AB isoform significantly attenuated TGF- $\beta$-mediated myofibroblast transformation and ECM gene expression. Our observations support previous findings that Hsp90 plays a critical role in activation of TGF- $\beta$ signaling, and inhibition of Hsp90 using 17-AAG blocks TGF- $\beta$-mediated fibroblast activation and ECM production by inhibiting the Smad and non-Smad pathways $(30,65)$. Taken together, these results suggest that Hsp90 is a central mediator for multiple signaling pathways and profibrotic processes that converge to activate fibroblasts in severe fibrotic disease.

In mammalian cells, 5 different isoforms of Hsp90 have been identified, and the Hsp90AA and Hsp$90 \mathrm{AB}$ isoforms are the 2 major members of the Hsp90 family. A gene duplication event was thought to 
be responsible for the generation of Hsp90AA and Hsp90AB isoforms, which share 85\% sequence identity and possess an ATPase catalytic center in their N-terminal domain $(45,46)$. However, structurally Hsp90AA lacks the signal peptide in its $\mathrm{N}$-terminus and possess a large variable region compared with the Hsp90AB isoform (44). Although Hsp90 was shown to interact with more than 700 client proteins and protein interactomes, Hsp90AA and Hsp90AB have unique and overlapping protein partners (45, 66-68). Notably, Hsp90AB interacts with a larger number of client proteins compared with Hsp90AA (45). Additionally, different isoforms of Hsp90 display distinct binding preferences for small-molecule inhibitors (69, 70). Our data reveal that the genetic knockdown of Hsp90AB, but not Hsp90AA, attenuates fibroproliferation, myofibroblast transformation, and ECM production. Further, we have identified several Hsp90-driven gene targets (CDK4, IGF1, MYCN, and SPHK1) that might play a critical role in fibroblast proliferation in severe fibrotic lung disease. The knockdown of Hsp90AB attenuated gene expression of the ECM genes Col1 $\alpha$, Col5 $\alpha$, and $\alpha$ Sma, but had no effect on Fn1, suggesting that FN1 may not be a direct target of Hsp90AB. However, our results from 17-AAG treatment of fibroblasts suggest that Hsp90 also regulated Fn1 expression via unknown mechanisms. In support of our findings, the Hsp90AB isoform is upregulated in breast and lung cancer tissues and the expression levels of Hsp90AB are associated with lymphatic invasion and decreased survival (26, 71-74). Earlier structural studies have demonstrated that Asp93 in Hsp90 makes a direct hydrogen bond with the exocyclic N6 group of adenine of bound ATP/ADP nucleotide and site-directed mutagenesis of Asp88 to asparagine (D88N) in Hsp90AB resulted in the loss of ATP binding and ATPase activity $(23,24,75)$. In support of our findings, overexpression of a dominant-negative mutant of Hsp90 (D88N) abolished stress fiber formation and migration (75). Additionally, Hsp90AB is induced in response to TGF- $\beta$ in fibroblasts isolated from systemic sclerosis patients and mouse skin, and inhibition of Hsp90AB in vitro using 17-dimethylaminoethylamino-17-demethoxygeldanamycin (17-DMAG) was sufficient to attenuate collagen production (30). Together, these results suggest an isoform-specific role for Hsp90 and its ATPase activity in fibrotic lung diseases.

Migration and invasion through matrix by fibroblasts is central to the pathogenesis of various cancers (76) and fibrotic processes $(6,7)$. Increased migration of fibroblasts and the number of circulating fibrocytes has been shown to be associated with severity of the disease in patients with IPF and mouse models of pulmonary fibrosis $(15,43,77,78)$. The direct role of Hsp90 isoforms in fibroblast migration has not been previously studied. We demonstrate that both Hsp90AA and Hsp90AB isoforms play an important role in the migration of primary lung fibroblasts. Invasive capacity is the most important and targetable malignant feature of cancer. Hsp90 plays a key role in cellular motility in breast, colorectal, and prostate cancers and the vascular smooth-muscle cells of atherosclerosis, and inhibition of Hsp90 resulted in restriction of cellular migration and invasion (79). In agreement with this, our data demonstrate that Hsp90 plays an important role in fibroblast and fibrocyte migration and invasion in pulmonary fibrosis, since targeting Hsp90 with 17-AAG results in almost complete suppression of migration and invasion. We demonstrated that Hsp90 is a positive regulator of F-actin filament formation by modulating the levels of FAK, Cdc42, and phosphoERK. In particular, Hsp90 ATPase activity is required for Cdc42 production, but has no effect on expression of Rho, a family of small GTPases that are key molecular regulators of cell motility $(80,81)$. Binding of Rho, Rac, and Cdc42 to GTP results in recruitment of a range of target proteins that drive actin reorganization (81-83). RhoA promotes formation of actin stress fibers, whereas Cdc42 signaling leads to the formation of actin-rich spikes called filopodia (82). However, we did not observe changes in phosphorylation of FAK or total ERK levels, suggesting differential effects of Hsp90 on regulation of FAK and ERK signaling in fibroblast migration and invasiveness. In support of our observations in pulmonary fibrosis, reports from scleroderma and bleomycin mouse models of skin fibrosis suggest that elevated Hsp90 activates fibroblasts by stabilizing the TGF- $\beta$ receptor and integrin-linked kinase, which in turn modulate focal adhesion dynamics and fibroblast motility in skin fibrosis (84). These data suggest to us that inhibition of Hsp90 is a reasonable strategy for attenuating fibroblast motility in IPF. Further, activated fibroblasts isolated from fibrotic lesions express high levels of chemokines and chemokine receptors and secrete profibrotic cytokines, which are potential mediators of fibroblast migration and the fibrogenesis during tissue injury. The invasive phenotype of fibrotic fibroblasts induces expression of elevated chemokine receptors (e.g., CD44 and CCR5 and its corresponding ligands, such as hyaluronan) in response to the lung injury in $\operatorname{IPF}(6,7,41)$. Conditional deletion of HAS2 or targeting the CD44 receptor using antibodies was sufficient to inhibit fibroblast invasion and pulmonary fibrosis in bleomycin-induced pulmonary fibrosis $(7,41,85)$. Our new findings have identified Hsp90 as a promising candidate to inhibit CCR5, CD44, and HAS2 expression. Also, Hsp90 regulates 


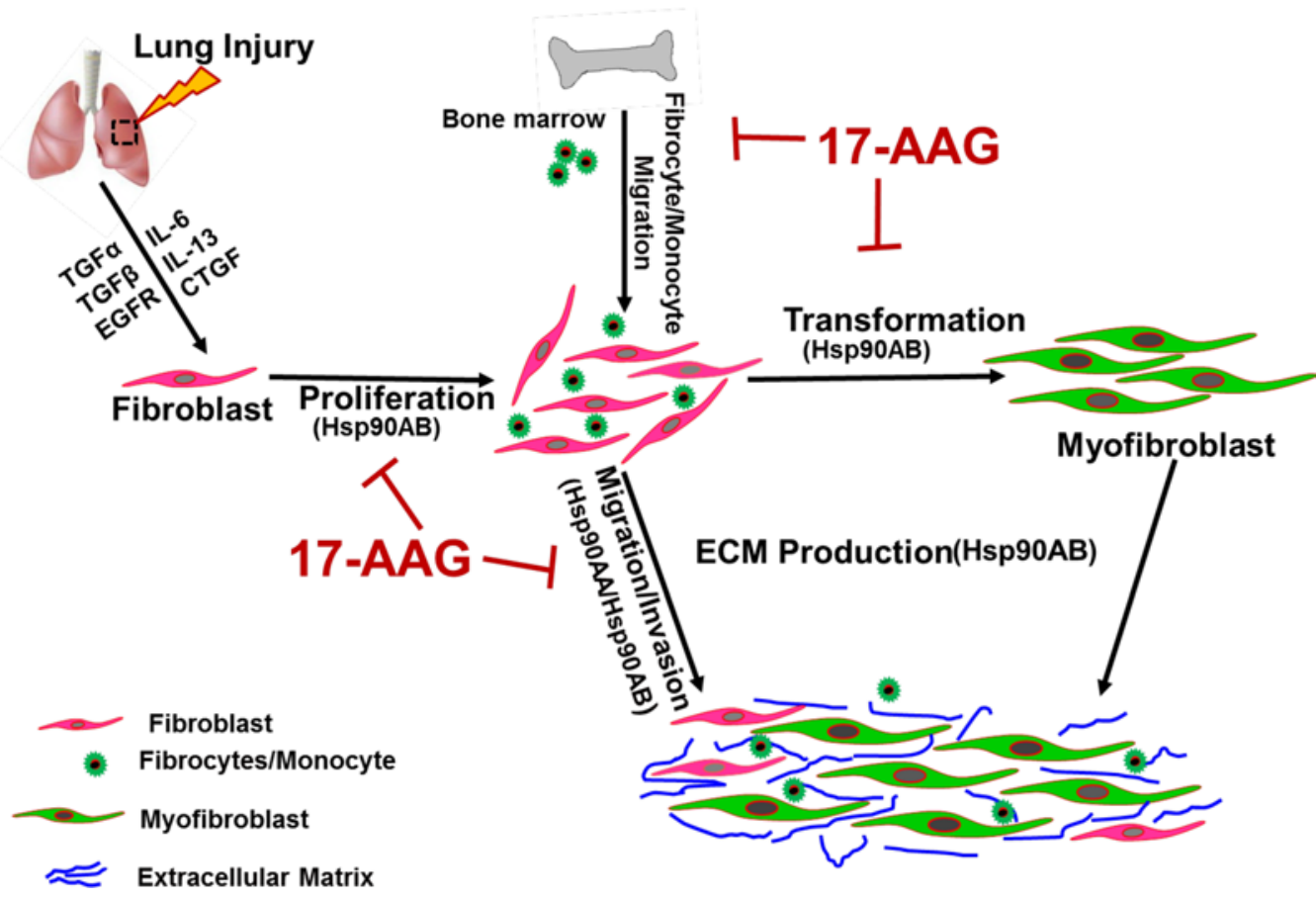

Figure 9. Schematic representation of Hsp90-regulated fibroblast functions in the pathogenesis of pulmonary fibrosis.

the expression of MMP12 and TIMP1, which have been shown to positively regulate fibroblast invasion (47). This property of Hsp90 further strengthens the rationale for the use of an Hsp90 inhibitor to prevent fibroblast invasion and pulmonary fibrosis.

Using immunostaining for Ki67, PCNA, and the BrdU-incorporation assay, we have demonstrated that inhibition of Hsp90 ATPase activity by 17-AAG attenuates proliferation of fibroblasts isolated from both IPF patients and TGF- $\alpha$-induced

pulmonary fibrosis in mice. Notably, both Ki67 staining and subpleural thickening were attenuated in TGF- $\alpha-T g$ mice treated with 17-AAG in vivo. Analysis of the dose-dependent kinetics for 17-AAG-mediated attenuation of fibroproliferation demonstrated that $50 \mathrm{nM}$ 17-AAG was sufficient to significantly inhibit activated fibroblast proliferation over 48 hours without detectable cell necrosis in vitro. Fibroblast proliferation is an important cellular process involved in pulmonary fibrogenesis, and IPF-directed therapies such as nintedanib act to attenuate the proliferation of lung fibroblasts and inhibit differentiation of fibroblasts into myofibroblasts (20). Several lines of evidence from cancer studies have shown that Hsp90 plays a critical role in cell proliferation by regulating progression through the G2/M cell-cycle phase (21, 60). Inhibition of Hsp90 by 17-AAG induces cell-cycle arrest and results in apoptosis of a lymphoma cell line through downregulation of cyclin D1 and Cdk4 and by activation of caspase 9 (86). Hsp90 inhibitors, such as STA-9090, inhibit proliferation and induce apoptosis in K-Ras-, HER2/neu-, and EGFR-mutant non-small-cell lung cancer cell lines (26), suggesting a role for Hsp90 in different signaling pathways that converge to cell proliferation. IGF1 and SPHK1 have been shown to be upregulated in response to TGF- $\beta$ stimuli in lung fibroblasts, and blockade of the above gene expression resulted in abrogation of fibroblast proliferation and reduced pulmonary fibrosis in the bleomycin-induced fibrosis mouse model (87-89). Similarly, MYCN, a known regulator of lung progenitor cell proliferation and differentiation during lung development, is upregulated in TGF- $\alpha$-induced pulmonary fibrosis $(15,90)$. Hsp90-specific IPF networks have identified CDK4, IGF1, SPHK1, and MYCN as potential gene targets that may regulate the proliferation of fibroblast in pulmonary fibrosis. We have demonstrated in vitro that inhibition of Hsp90 ATPase activity in fibroblasts downregulates these gene transcripts, thereby regulating fibroproliferation. However, Hsp90 has been shown to regulate several client proteins that might play an important role in the prosurvival of cancer cells. Hsp90-driven activation of antiapoptotic pathways may result in progressive expansion or impaired clearance of fibroblasts in subpleura and other areas of the lung $(25,91)$. Therefore, further studies are needed to identify the precise molecular interactions between the proliferative genes, survival genes, and Hsp90 in pulmonary fibrosis.

We conclude that increased Hsp90 ATPase activity plays a critical role in fibroblast activation in severe fibrotic lung disease. Gene-knockdown studies have demonstrated in vitro that Hsp90AB, but not Hsp90AA, is required for fibroblast proliferation, myofibroblast transformation, and ECM production, whereas the expression of both Hsp90AA and Hsp90AB isoforms was required in fibroblast migration. The findings from this study indicate that both Hsp90AA and Hsp90AB isoforms must be targeted to inhibit both overlapping and unique cellular properties by the isoforms (Figure 9). Our preclinical findings support a pathogenic 
role for Hsp90 in IPF and demonstrate that Hsp90 is a plausible therapeutic target at the molecular, cellular, and whole-animal levels. Our observations that Hsp90 inhibition attenuated fibroblast activation and myofibroblast accumulation and collagen deposition without any detectable toxic effects serve as proof of principle for a future interventional role of Hsp90 in the treatment of fibrotic lung disease.

\section{Methods}

Please see Supplemental material for methods not described in this section.

TGF- $\alpha$-transgenic mice and pharmacological treatment. TGF- $\alpha$-overexpressing mice were generated in an FVB/NJ inbred strain background as described previously (54). Homozygous Club cell (Clara cell)-specific protein-rtTA1/2 (CCSP) mice were mated with heterozygous (TetO) 7-cmv TGF- $\alpha-\mathrm{Tg}$ mice to generate transgenic TGF- $\alpha$ (CCSP/TGF- $\alpha$ ) mice. Female mice, 8-14 weeks old, were used in all experiments. Mice were housed under specific pathogen-free conditions, and all animal experimental protocols were approved by the IACUC of the Cincinnati Children's Hospital Research Foundation. TGF- $\alpha$ expression was induced by administering food containing Dox $(62.5 \mathrm{mg} / \mathrm{kg})$ to CCSP/TGF- $\alpha-\mathrm{Tg}$ mice for 6 weeks. CCSP $/-$ mice fed with Dox food were used as nonfibrotic controls. A stock solution of 17-AAG (Tocris) was prepared in $20 \%$ DMSO $+20 \%$ Cremophor $+60 \%$ PBS solution to make a $1 \mathrm{mg} / \mathrm{ml}$ drug solution. The drug was prepared every day, and dosing throughout the study was based on original baseline animal body weights and not adjusted for weight changes. Mice were treated with 17-AAG (15 mg/kg once daily, $7 \mathrm{~d} / \mathrm{wk}$ ) i.p. for 3 weeks. Control mice were treated with similar volumes of the vehicle. Mice were weighed at the beginning of the study and at weekly intervals.

aSMA reporter mice. The SMMHC ${ }^{\mathrm{CreERT} /+} \mathrm{ROSA}^{\mathrm{mTmG} /+}$ heterozygous male transgenic mouse strain was generated by crossing male SMMHC CreERT2/+ (Jackson Laboratory, stock 019079) mice with ROSA ${ }^{\mathrm{mTmC}}$ mice (15). Heterozygous SMMHC CreERT2/+ mice (knock-in allele) are phenotypically normal and can be used to track the genetic lineage of $\alpha$ SMA-expressing cells. Heterozygous SMMHC ${ }^{\mathrm{CreERT} /+} \mathrm{ROSA}^{\mathrm{mTmG} /+}$ mice (knock-in allele) express CreERT2 under the control of the promoter for mouse smooth muscle myosin, heavy polypeptide 11 (92).

Human tissue samples. Human IPF $(n=4)$ and control non-IPF $(n=6)$ biopsies were obtained from Eric White, Department of Internal Medicine, University of Michigan Health System (UMHS). The biopsy material consisted of archived, paraffin-embedded, pathological specimens previously acquired from adult patients who had undergone lung transplantation for IPF. IPF was diagnosed according to the American Thoracic Society consensus criteria (93). Lung samples from donors' lungs with no lung disease were used as normal lung biopsies. All tissues were acquired using research protocols and informed consent and approved by the UMHS Institutional Review Board. All materials were de-identified to the research team.

Computational analysis. A previously published transcriptomic data set (GSE53845) (8) derived from analysis of the lung biopsies of 40 IPF patients and 8 healthy controls and available in the National Center for Biotechnology Information (NCBI) Gene Expression Omnibus (GEO) (94) was used to identify DEGs. Differential analysis for genes was performed using the R package limma or GEO2R (http://www.ncbi. nlm.nih.gov/geo/geo2r/) with $P$ value and FDR threshold at 0.05 and fold-change threshold at 1.5 (95). This IPF gene signature was queried against the LINCS database (http://www.lincscloud.org/), a massive catalog of gene-expression profiles collected from human cells treated with chemical and genetic perturbagens, using a connectivity-map approach as described previously (38). Briefly, the IPF DEG signature is queried against the 1.4 million gene-expression profiles in the LINCS L1000 dataset. The pattern-matching software in the LINCS cloud searches for 2-directional matches, taking into account both the up and down gene sets, in comparing IPF query against L1000 compound signatures ( $Z$-scored differential expressions). The LINCScloud query app (http://apps.lincscloud.org/) then generates a list of rank-ordered signatures based on their strength of the match to the query, from highest to lowest. Functional enrichment analysis of the negatively correlated gene sets between IPF lungs and 17-AAG-treated cells from the LINCS database was conducted using the ToppFun application of the ToppGene Suite (39). For network representation of select significantly enriched biological processes and pathways, we used Cytoscape (96).

Immunohistochemistry. Formalin- or OCT-fixed human lung tissue sections from non-IPF $(n=3)$ and IPF $(n=4)$ patients were prepared and immunostained with antibodies against Hsp90 (Santa Cruz Biotechnology, SC-7947) and vimentin (Abcam, ab137321) as described previously (15). All images were obtained using a Leica DM2700 M bright-field microscope. High-magnification images $(\times 40)$ were captured with a $3 \mathrm{CCD}$ color video camera. 
Histology, pleural thickness, and chord length measurement. Lungs were inflation fixed using 10\% formalin and stained with Masson's trichrome or H\&E as previously described (15). For pleural thickness measurement, 5 random measures per lung section were obtained for each animal using a brightfield microscope (Leica Microsystems). For chord length measurements, 7 randomly selected uniform fields per lung tissue section in the alveolar regions were obtained for each animal. High-magnification images $(\times 40)$ were captured with a 3 CCD color video camera and analyzed using MetaMorph imaging software (v6.2; Molecular Devices). Pleural thickness and mean chord length were measured using the measured-distance function of MetaMorph.

Hydroxyproline assay. Lung fibrosis was assessed by measuring hydroxyproline levels and normalizing to lung weight, using a colorimetric assay as previously described $(6,97)$.

Statistics. All data were analyzed using Prism (version 5; GraphPad). One-way ANOVA with Sidak's multiple comparison post-test was used to compare different experimental groups. Student's $t$ test was used to compare between 2 experimental groups. Data were considered statistically significant for $P$ values less than 0.05 .

\section{Author contributions}

SKM, VS, and AGJ conceived the project, designed experiments, analyzed data and wrote the manuscript. Figure 1, Table 1, and Supplemental Tables 1 and 2 were produced by AGJ and YW. Figure 2 was produced by VS and ESW. Figures 2-9 were produced by VS, GBR, APN, FXM, and SKM. Figures 3C and $3 \mathrm{D}$ were produced by SKM. Figure $8 \mathrm{~F}$ was produced by RKK.

\section{Acknowledgments}

The authors thank the veterinary services and pathology research core at Cincinnati Children's Hospital Medical Center for the help in this study. We thank William D. Hardie and Shiva K. Shanmukhappa for valuable suggestions. This research was supported by the NIH grant NHLBI 1R21HL133539 (to SKM and AGJ), a Parker B. Francis Fellowship (to SKM), and Council of Scientific and Industrial Research Fellowship, Government of India (to VS). ESW is supported in part by the University of Michigan Human Lung Biorepository Fund.

Address correspondence to: Satish K. Madala, Division of Pulmonary Medicine, Cincinnati Children's Hospital Medical Center, 3333 Burnet Avenue, MLC 2021, Cincinnati, Ohio 45229, USA. Phone: 513.636.9852; E-mail: satish.madala@cchmc.org. Or to: Anil G. Jegga, Division of Biomedical informatics, Cincinnati Children's Hospital Medical Center, 240 Albert Sabin Way, MLC 7024, Cincinnati, Ohio 45229, USA. Phone: 513.636.0261; E-mail: anil.jegga@cchmc.org.

1. Wynn TA, Ramalingam TR. Mechanisms of fibrosis: therapeutic translation for fibrotic disease. Nat Med. 2012;18(7):1028-1040.

2. Glasser SW, Hagood JS, Wong S, Taype CA, Madala SK, Hardie WD. Mechanisms of lung fibrosis resolution. Am J Pathol. 2016;186(5):1066-1077.

3. du Bois RM, et al. Ascertainment of individual risk of mortality for patients with idiopathic pulmonary fibrosis. Am J Respir Crit Care Med. 2011;184(4):459-466.

4. Blackwell TS, et al. Future directions in idiopathic pulmonary fibrosis research. An NHLBI workshop report. Am J Respir Crit Care Med. 2014;189(2):214-222.

5. Xia H, et al. Identification of a cell-of-origin for fibroblasts comprising the fibrotic reticulum in idiopathic pulmonary fibrosis. Am J Pathol. 2014;184(5):1369-1383.

6. Madala SK, Edukulla R, Schmidt S, Davidson C, Ikegami M, Hardie WD. Bone marrow-derived stromal cells are invasive and hyperproliferative and alter transforming growth factor- $\alpha$-induced pulmonary fibrosis. Am J Respir Cell Mol Biol. 2014;50(4):777-786.

7. Li Y, et al. Severe lung fibrosis requires an invasive fibroblast phenotype regulated by hyaluronan and CD44. J Exp Med. 2011;208(7):1459-1471.

8. DePianto DJ, et al. Heterogeneous gene expression signatures correspond to distinct lung pathologies and biomarkers of disease severity in idiopathic pulmonary fibrosis. Thorax. 2015;70(1):48-56.

9. Zoz DF, Lawson WE, Blackwell TS. Idiopathic pulmonary fibrosis: a disorder of epithelial cell dysfunction. Am JMed Sci. 2011;341(6):435-438

10. Kropski JA, et al. Extensive phenotyping of individuals at risk for familial interstitial pneumonia reveals clues to the pathogenesis of interstitial lung disease. Am J Respir Crit Care Med. 2015;191(4):417-426.

11. White ES, Mantovani AR. Inflammation, wound repair, and fibrosis: reassessing the spectrum of tissue injury and resolution. J Pathol. 2013;229(2):141-144.

12. Steele MP, Schwartz DA. Molecular mechanisms in progressive idiopathic pulmonary fibrosis. Annu Rev Med. 2013;64:265-276.

13. Thannickal VJ, Toews GB, White ES, Lynch JP, Martinez FJ. Mechanisms of pulmonary fibrosis. Annu Rev Med. 2004;55:395-417. 
14. Hinz B, et al. Recent developments in myofibroblast biology: paradigms for connective tissue remodeling. Am J Pathol. 2012;180(4):1340-1355.

15. Sontake V, et al. Fibrocytes regulate Wilms tumor 1-positive cell accumulation in severe fibrotic lung disease. J Immunol. 2015;195(8):3978-3991.

16. Hardie WD, et al. Signaling pathways in the epithelial origins of pulmonary fibrosis. Cell Cycle. 2010;9(14):2769-2776.

17. Madala SK, et al. p70 ribosomal S6 kinase regulates subpleural fibrosis following transforming growth factor- $\alpha$ expression in the lung. Am J Physiol Lung Cell Mol Physiol. 2016;310(2):L175-L186.

18. Madala SK, et al. Dual targeting of MEK and PI3K pathways attenuates established and progressive pulmonary fibrosis. PLoS ONE. 2014;9(1):e86536.

19. Madala SK, Schmidt S, Davidson C, Ikegami M, Wert S, Hardie WD. MEK-ERK pathway modulation ameliorates pulmonary fibrosis associated with epidermal growth factor receptor activation. Am J Respir Cell Mol Biol. 2012;46(3):380-388.

20. Wollin L, et al. Mode of action of nintedanib in the treatment of idiopathic pulmonary fibrosis. Eur Respir J. 2015;45(5):1434-1445.

21. Taipale M, Jarosz DF, Lindquist S. HSP90 at the hub of protein homeostasis: emerging mechanistic insights. Nat Rev Mol Cell Biol. 2010;11(7):515-528.

22. Schwenkert S, Hugel T, Cox MB. The Hsp90 ensemble: coordinated Hsp90-cochaperone complexes regulate diverse cellular processes. Nat Struct Mol Biol. 2014;21(12):1017-1021.

23. Obermann WM, Sondermann H, Russo AA, Pavletich NP, Hartl FU. In vivo function of Hsp90 is dependent on ATP binding and ATP hydrolysis. J Cell Biol. 1998;143(4):901-910.

24. Panaretou B, et al. ATP binding and hydrolysis are essential to the function of the Hsp 90 molecular chaperone in vivo. $E M B O J$. 1998;17(16):4829-4836.

25. Trepel J, Mollapour M, Giaccone G, Neckers L. Targeting the dynamic HSP90 complex in cancer. Nat Rev Cancer. 2010;10(8):537-549

26. Wang Y, Trepel JB, Neckers LM, Giaccone G. STA-9090, a small-molecule Hsp90 inhibitor for the potential treatment of cancer. Curr Opin Investig Drugs. 2010;11(12):1466-1476.

27. Kim T, Keum G, Pae AN. Discovery and development of heat shock protein 90 inhibitors as anticancer agents: a review of patented potent geldanamycin derivatives. Expert Opin Ther Pat. 2013;23(8):919-943.

28. Czar MJ, Welsh MJ, Pratt WB. Immunofluorescence localization of the 90-kDa heat-shock protein to cytoskeleton. Eur J Cell Biol. 1996;70(4):322-330.

29. Harlow L, et al. Identification of citrullinated hsp90 isoforms as novel autoantigens in rheumatoid arthritis-associated interstitial lung disease. Arthritis Rheum. 2013;65(4):869-879.

30. Tomcik M, et al. Heat shock protein 90 (Hsp90) inhibition targets canonical TGF- $\beta$ signalling to prevent fibrosis. Ann Rheum Dis. 2014;73(6):1215-1222.

31. Datta R, et al. Hsp90/Cdc37 assembly modulates TGF $\beta$ receptor-II to act as a profibrotic regulator of TGF $\beta$ signaling during cardiac hypertrophy. Cell Signal. 2015;27(12):2410-2424.

32. Bhagwat N, et al. Improved targeting of JAK2 leads to increased therapeutic efficacy in myeloproliferative neoplasms. Blood. 2014;123(13):2075-2083.

33. Enmon R, et al. Combination treatment with 17-N-allylamino-17-demethoxy geldanamycin and acute irradiation produces supra-additive growth suppression in human prostate carcinoma spheroids. Cancer Res. 2003;63(23):8393-8399.

34. Yun IS, Lee MH, Rah DK, Lew DH, Park JC, Lee WJ. Heat shock protein 90 inhibitor (17-AAG) induces apoptosis and decreases cell migration/motility of keloid fibroblasts. Plast Reconstr Surg. 2015;136(1):44e-53e.

35. Noh H, et al. Heat shock protein 90 inhibitor attenuates renal fibrosis through degradation of transforming growth factor- $\beta$ type II receptor. Lab Invest. 2012;92(11):1583-1596.

36. Myung SJ, Yoon JH, Kim BH, Lee JH, Jung EU, Lee HS. Heat shock protein 90 inhibitor induces apoptosis and attenuates activation of hepatic stellate cells. J Pharmacol Exp Ther. 2009;330(1):276-282.

37. Sun X, Zhang XD, Cheng G, Hu YH, Wang HY. Inhibition of hepatic stellate cell proliferation by heat shock protein 90 inhibitors in vitro. Mol Cell Biochem. 2009;330(1-2):181-185.

38. Lamb J, et al. The Connectivity Map: using gene-expression signatures to connect small molecules, genes, and disease. Science. 2006;313(5795):1929-1935.

39. Chen J, Bardes EE, Aronow BJ, Jegga AG. ToppGene Suite for gene list enrichment analysis and candidate gene prioritization. Nucleic Acids Res. 2009;37(Web Server issue):W305-W311.

40. Kamal A, et al. A high-affinity conformation of Hsp90 confers tumour selectivity on Hsp90 inhibitors. Nature. 2003;425(6956):407-410.

41. Xie T, et al. Transcription factor TBX4 regulates myofibroblast accumulation and lung fibrosis. J Clin Invest. 2016;126(8):3063-3079.

42. Fujiwara A, et al. Correlation between circulating fibrocytes, and activity and progression of interstitial lung diseases. Respirology. 2012;17(4):693-698.

43. Moeller A, et al. Circulating fibrocytes are an indicator of poor prognosis in idiopathic pulmonary fibrosis. Am J Respir Crit Care Med. 2009;179(7):588-594.

44. Chen B, Zhong D, Monteiro A. Comparative genomics and evolution of the HSP90 family of genes across all kingdoms of organisms. BMC Genomics. 2006;7:156.

45. Zuehlke AD, Beebe K, Neckers L, Prince T. Regulation and function of the human HSP90AA1 gene. Gene. 2015;570(1):8-16.

46. Haase M, Fitze G. HSP90AB1: Helping the good and the bad. Gene. 2016;575(2 Pt 1):171-186.

47. Madala SK, et al. Matrix metalloproteinase 12-deficiency augments extracellular matrix degrading metalloproteinases and attenuates IL-13-dependent fibrosis. J Immunol. 2010;184(7):3955-3963.

48. Barbarin V, Xing Z, Delos M, Lison D, Huaux F. Pulmonary overexpression of IL-10 augments lung fibrosis and Th2 responses induced by silica particles. Am J Physiol Lung Cell Mol Physiol. 2005;288(5):L841-L848.

49. Hinz B, Phan SH, Thannickal VJ, Galli A, Bochaton-Piallat ML, Gabbiani G. The myofibroblast: one function, multiple origins. Am J Pathol. 2007;170(6):1807-1816.

50. Kis K, Liu X, Hagood JS. Myofibroblast differentiation and survival in fibrotic disease. Expert Rev Mol Med. 2011;13:e27. 
51. Klingberg F, Hinz B, White ES. The myofibroblast matrix: implications for tissue repair and fibrosis. J Pathol. 2013;229(2):298-309.

52. Sheppard D. Transforming growth factor beta: a central modulator of pulmonary and airway inflammation and fibrosis. Proc Am Thorac Soc. 2006;3(5):413-417.

53. Decologne N, et al. TGF-betal induces progressive pleural scarring and subpleural fibrosis. J Immunol. 2007;179(9):6043-6051.

54. Hardie WD, Le Cras TD, Jiang K, Tichelaar JW, Azhar M, Korfhagen TR. Conditional expression of transforming growth factor-alpha in adult mouse lung causes pulmonary fibrosis. Am J Physiol Lung Cell Mol Physiol. 2004;286(4):L741-L749.

55. Hardie WD, et al. Genomic profile of matrix and vasculature remodeling in TGF-alpha induced pulmonary fibrosis. Am J Respir Cell Mol Biol. 2007;37(3):309-321.

56. Bellaye PS, Burgy O, Causse S, Garrido C, Bonniaud P. Heat shock proteins in fibrosis and wound healing: good or evil? Phar macol Ther. 2014;143(2):119-132.

57. Cool CD, Groshong SD, Rai PR, Henson PM, Stewart JS, Brown KK. Fibroblast foci are not discrete sites of lung injury or repair: the fibroblast reticulum. Am J Respir Crit Care Med. 2006;174(6):654-658.

58. Waza M, et al. 17-AAG, an Hsp90 inhibitor, ameliorates polyglutamine-mediated motor neuron degeneration. Nat Med. 2005;11(10):1088-1095.

59. Ho SW, et al. Effects of 17-allylamino-17-demethoxygeldanamycin (17-AAG) in transgenic mouse models of frontotemporal lobar degeneration and Alzheimer's disease. Transl Neurodegener. 2013;2(1):24.

60. Pillai RN, Ramalingam SS. Heat shock protein 90 inhibitors in non-small-cell lung cancer. Curr Opin Oncol. 2014;26(2):159-164.

61. Pohlers D, et al. TGF-beta and fibrosis in different organs - molecular pathway imprints. Biochim Biophys Acta. 2009;1792(8):746-756.

62. Branton MH, Kopp JB. TGF-beta and fibrosis. Microbes Infect. 1999;1(15):1349-1365.

63. Meng XM, Nikolic-Paterson DJ, Lan HY. TGF- $\beta$ : the master regulator of fibrosis. Nat Rev Nephrol. 2016;12(6):325-338.

64. Madala SK, et al. Inhibition of the $\alpha \mathrm{v} \beta 6$ integrin leads to limited alteration of TGF- $\alpha$-induced pulmonary fibrosis. Am J Physiol Lung Cell Mol Physiol. 2014;306(8):L726-L735.

65. Wrighton KH, Lin X, Feng XH. Critical regulation of TGFbeta signaling by Hsp90. Proc Natl Acad Sci USA. 2008;105(27):9244-9249.

66. Buchner J. Hsp90 \& Co. - a holding for folding. Trends Biochem Sci. 1999;24(4):136-141.

67. Pearl LH, Prodromou C. Structure and mechanism of the Hsp90 molecular chaperone machinery. Annu Rev Biochem. 2006; 75:271-294

68. Picard D. Heat-shock protein 90, a chaperone for folding and regulation. Cell Mol Life Sci. 2002;59(10):1640-1648

69. Prince TL, et al. Client proteins and small molecule inhibitors display distinct binding preferences for constitutive and stressinduced HSP90 isoforms and their conformationally restricted mutants. PLoS ONE. 2015;10(10):e0141786.

70. Patel PD, et al. Paralog-selective Hsp90 inhibitors define tumor-specific regulation of HER2. Nat Chem Biol. 2013;9(11):677-684.

71. Biaoxue R, et al. Upregulation of Hsp90-beta and annexin A1 correlates with poor survival and lymphatic metastasis in lung cancer patients. J Exp Clin Cancer Res. 2012;31:70.

72. Bagatell R, Khan O, Paine-Murrieta G, Taylor CW, Akinaga S, Whitesell L. Destabilization of steroid receptors by heat shock protein 90-binding drugs: a ligand-independent approach to hormonal therapy of breast cancer. Clin Cancer Res. 2001;7(7):2076-2084.

73. Jego G, Hazoumé A, Seigneuric R, Garrido C. Targeting heat shock proteins in cancer. Cancer Lett. 2013;332(2):275-285.

74. Rong B, et al. Identification and verification of Hsp90-beta as a potential serum biomarker for lung cancer. Am J Cancer Res. 2014;4(6):874-885.

75. Miao RQ, Fontana J, Fulton D, Lin MI, Harrison KD, Sessa WC. Dominant-negative Hsp90 reduces VEGF-stimulated nitric oxide release and migration in endothelial cells. Arterioscler Thromb Vasc Biol. 2008;28(1):105-111.

76. Kalluri R, Zeisberg M. Fibroblasts in cancer. Nat Rev Cancer. 2006;6(5):392-401.

77. Moore BB, et al. CCR2-mediated recruitment of fibrocytes to the alveolar space after fibrotic injury. Am J Pathol. 2005;166(3):675-684.

78. Bellini A, Mattoli S. The role of the fibrocyte, a bone marrow-derived mesenchymal progenitor, in reactive and reparative fibroses. Lab Invest. 2007;87(9):858-870.

79. Kim J, Jang SW, Park E, Oh M, Park S, Ko J. The role of heat shock protein 90 in migration and proliferation of vascular smooth muscle cells in the development of atherosclerosis. J Mol Cell Cardiol. 2014;72:157-167.

80. Ridley AJ. Rho GTPases and cell migration. J Cell Sci. 2001;114(Pt 15):2713-2722.

81. Amano M, et al. Formation of actin stress fibers and focal adhesions enhanced by Rho-kinase. Science. 1997;275(5304):1308-1311.

82. Ridley AJ, Paterson HF, Johnston CL, Diekmann D, Hall A. The small GTP-binding protein rac regulates growth factorinduced membrane ruffling. Cell. 1992;70(3):401-410.

83. Kimura K, et al. Regulation of myosin phosphatase by Rho and Rho-associated kinase (Rho-kinase). Science. 1996;273(5272):245-248.

84. Radovanac K, et al. Stabilization of integrin-linked kinase by the Hsp90-CHIP axis impacts cellular force generation, migration and the fibrotic response. EMBO J. 2013;32(10):1409-1424.

85. Phillips RJ, et al. Circulating fibrocytes traffic to the lungs in response to CXCL12 and mediate fibrosis. J Clin Invest. 2004;114(3):438-446.

86. Georgakis GV, Li Y, Younes A. The heat shock protein 90 inhibitor 17-AAG induces cell cycle arrest and apoptosis in mantle cell lymphoma cell lines by depleting cyclin D1, Akt, Bid and activating caspase 9. Br J Haematol. 2006;135(1):68-71.

87. Yamanaka M, et al. Sphingosine kinase 1 (SPHK1) is induced by transforming growth factor-beta and mediates TIMP-1 upregulation. J Biol Chem. 2004;279(52):53994-54001.

88. Hung CF, Rohani MG, Lee SS, Chen P, Schnapp LM. Role of IGF-1 pathway in lung fibroblast activation. Respir Res. 2013;14:102.

89. Kim TH, Chow YH, Gill SE, Schnapp LM. Effect of insulin-like growth factor blockade on hyperoxia-induced lung injury. Am J Respir Cell Mol Biol. 2012;47(3):372-378.

90. Okubo T, Knoepfler PS, Eisenman RN, Hogan BL. Nmyc plays an essential role during lung development as a dosage-sensitive regulator of progenitor cell proliferation and differentiation. Development. 2005;132(6):1363-1374. 
91. Chakraborty A, et al. HSP90 regulates cell survival via inositol hexakisphosphate kinase-2. Proc Natl Acad Sci USA. 2008;105(4):1134-1139.

92. Wirth A, et al. G12-G13-LARG-mediated signaling in vascular smooth muscle is required for salt-induced hypertension. Nat Med. 2008;14(1):64-68.

93. American Thoracic Society. Idiopathic pulmonary fibrosis: diagnosis and treatment. International consensus statement. American Thoracic Society (ATS), and the European Respiratory Society (ERS). Am J Respir Crit Care Med. 2000;161(2 Pt 1):646-664.

94. Barrett T, et al. NCBI GEO: mining tens of millions of expression profiles--database and tools update. Nucleic Acids Res. 2007;35(Database issue):D760-D765.

95. Ritchie ME, et al. limma powers differential expression analyses for RNA-sequencing and microarray studies. Nucleic Acids Res. 2015;43(7):e47.

96. Shannon P, et al. Cytoscape: a software environment for integrated models of biomolecular interaction networks. Genome Res. 2003;13(11):2498-2504

97. Wynn TA, et al. Quantitative assessment of macrophage functions in repair and fibrosis. Curr Protoc Immunol. 2011; Chapter 14:Unit14.22. 